\title{
Graßmannian integrals in Minkowski signature, amplitudes, and integrability
}

\author{
Nils Kanning ${ }^{a, b}$ and Matthias Staudacher ${ }^{a}$ \\ ${ }^{a}$ Institut für Mathematik und Institut für Physik, Humboldt-Universität zu Berlin, \\ IRIS-Adlershof, Zum Großen Windkanal 6, 12489 Berlin, Germany \\ ${ }^{b}$ Arnold Sommerfeld Center for Theoretical Physics, Ludwig-Maximilians-Universität, \\ Theresienstraße 37, 80333 München, Germany ${ }^{1}$ \\ E-mail: kanning@physik.hu-berlin.de, staudacher@physik.hu-berlin.de
}

ABSTRACT: We attempt to systematically derive tree-level scattering amplitudes in fourdimensional, planar, maximally supersymmetric Yang-Mills theory from integrability. We first review the connections between integrable spin chains, Yangian invariance, and the construction of such invariants in terms of Graßmannian contour integrals. Building upon these results, we equip a class of Graßmannian integrals for general symmetry algebras with unitary integration contours. These contours emerge naturally by paying special attention to the proper reality conditions of the algebras. Specializing to $\mathfrak{p s u}(2,2 \mid 4)$ and thus to maximal superconformal symmetry in Minkowski space, we find in a number of examples expressions similar to, but subtly different from the perturbative physical scattering amplitudes. Our results suggest a subtle breaking of Yangian invariance for the latter, with curious implications for their construction from integrability.

Keywords: Lattice Integrable Models, Matrix Models, Scattering Amplitudes, Supersymmetric Gauge Theory

ArXiv ePrint: 1811.04949

\footnotetext{
${ }^{1}$ Address until January 2018.
} 


\section{Contents}

1 Introduction $\quad 2$

2 Review: amplitudes and symmetries 2

2.1 Amplitudes in spinor helicity variables 3

2.2 Yangian symmetry 4

$\begin{array}{lll}2.3 & \text { Graßmannian integral and deformations } & 7\end{array}$

3 Unitary Graßmannian integral $\quad 10$

$\begin{array}{lll}3.1 & \text { Reality conditions and unitary contour } & 10\end{array}$

$\begin{array}{lll}3.2 & \text { Graßmannian integral } & 11\end{array}$

$\begin{array}{ll}3.3 & \text { Single-valued integrand } \\ 3.4\end{array}$

$\begin{array}{lll}3.4 & \text { Symmetry generators and Yangian invariance } & 14\end{array}$

4 Sample invariants and amplitudes $\quad \mathbf{1 5}$

$\begin{array}{lll}4.1 & \text { Four particles for } \mathfrak{u}(2,2 \mid 4) & 16\end{array}$

4.2 Six particles for $\mathfrak{u}(2,2 \mid 4) \quad 16$

$\begin{array}{lll}4.3 \text { Toy model and implications: four particles for } \mathfrak{u}(1,1) & 19\end{array}$

4.4 Eight particles for $\mathfrak{u}(2,2 \mid 4) \quad 21$

5 Graßmannian integral in oscillator basis 24

5.1 Oscillator representations 24

$\begin{array}{ll}5.2 \text { Graßmannian integral } 25 & 25\end{array}$

$\begin{array}{lll}5.3 & \text { Bargmann realization and transformation } & 27\end{array}$

5.4 Transformation of generators and integral 29

$6 \quad$ R-matrix and divergencies $\quad 31$

6.1 R-matrices of Heisenberg chain and spectral problem 31

6.2 Normalization and divergent terms 33

$\begin{array}{lll}7 & \text { Link to cusp equation } & 36\end{array}$

$\begin{array}{lll}8 & \text { Conclusions and outlook } & 37\end{array}$

$\begin{array}{ll}\text { A Parity symmetry } & 38\end{array}$

B Unitary contour from gluing $\quad 39$ 


\section{Introduction}

Four-dimensional, planar, maximally supersymmetric Yang-Mills theory is surely integrable at generic 't Hooft coupling, even though there still is no precise formulation, let alone a completed proof, of this statement. A presumably related problem is the absence of a systematic derivation procedure for generic quantities from "integrability" in this $\mathcal{N}=4$ SYM model. Once established, one would ideally like to start from the underlying (algebraic?) integrable structure, and subsequently systematically derive non-perturbatively all quantities one fancies from a single principle: spectrum, states, correlation functions, Wilson loops, scattering amplitudes, form factors, etc. In practice, it is of course unlikely that one will always succeed in completely "solving" for a given quantity of interest. But at least one would like to know where to start from.

This lack of a solid starting point is particularly vexing in the case of the scattering amplitudes of the model. Used by skillful hands, the magic integrability machine yields, in certain kinematical limits and employing various assumptions, highly non-trivial analytical results for strong coupling, for an impressive number of loops at weak coupling, and even, in some special cases, for generic coupling. What then, precisely, is the underlying symmetry or principle for these successes? It has been known now for nearly a decade that the infinitesimal superconformal symmetry of tree-level amplitudes combines with a distinct second, dual superconformal copy into a Yangian symmetry, i.e. into the mathematical structure underlying (rational) integrable spin chains. But, in contradistinction to the case of spin chains, it has not yet been possible to turn this around, and to derive the tree-level amplitudes from this symmetry. The present study initially set out to fill this gap. However, as we will see below, this is far less straightforward than one might have suspected. In fact, we shall find subtle differences between the results obtained from Yangian invariance and the physical amplitudes. We will discuss possible consequences of these surprising results in our conclusion section 8 below.

\section{Review: amplitudes and symmetries}

We begin with a short review of tree-level scattering amplitudes in planar $\mathcal{N}=4$ SYM theory. Naturally, we concentrate on those aspects that form the foundation for our own work presented in this paper, which is in part based on the PhD thesis [1] of one of the authors. In particular, we introduce spinor helicity variables and highlight the reality conditions of the particle momenta. Moreover, we discuss the integrable structure of the amplitudes, which is closely tied to an infinite-dimensional Yangian symmetry extending their superconformal symmetry. We recapitulate a formulation of the amplitudes as Graßmannian integrals, which conveniently exposes these symmetries. This formulation led to a proposal for deformations of the amplitudes preserving Yangian symmetry, which we also review. More extensive surveys of gauge theory scattering amplitudes can be found in [2-4] and special properties in case of the $\mathcal{N}=4$ theory are discussed e.g. in $[5,6]$. 


\subsection{Amplitudes in spinor helicity variables}

A scattering process in planar $\mathcal{N}=4$ SYM theory involving the particles $i=1, \ldots, N$ is characterized by their null momenta $p^{i} \in \mathbb{R}^{1,3}$ and helicities $h^{i}=-1,-\frac{1}{2}, 0,+\frac{1}{2},+1$, whose range is determined by the internal $\mathfrak{s u}(4) \mathrm{R}$-symmetry. In addition, it depends on color information originating from the gauge group $\mathrm{SU}\left(N_{\mathrm{C}}\right)$. However, this color structure can easily be stripped off from the scattering amplitude. The total momentum is conserved in the scattering process, $P=\sum_{i} p^{i}=0$. In general, this is not true for the total helicity $\sum_{i} h^{i}$. The introduction of fermionic variables $\tilde{\eta}_{\dot{a}}^{i}$ with $\dot{a}=1,2,3,4$ allows to package all amplitudes with a common degree of helicity violation $2 K=N-\sum_{i} h^{i}$ into a single superamplitude. In the following, we are dealing with color-stripped tree-level superamplitudes $\mathcal{A}_{N, K}$, which are functions of the momenta $p^{i}$ and the fermions $\tilde{\eta}^{i}$. Mostly, we refer to them simply as amplitudes.

The $\mathcal{A}_{N, K}$ can in principle be constructed employing the textbook Feynman diagram approach. In practice, however, this is almost infeasible because the number of diagrams grows exceedingly fast with the number of particles $N$, see e.g. the discussion in [7]. In addition, the computation of individual diagrams completely obscures an unexpected simplicity in the expressions for the complete amplitudes. To uncover this simplicity, we have to choose suitable variables for the kinematics. This is achieved by spinor helicity variables for the particle momenta $p^{i}$, which date back to the 1920s [8, 9]. To introduce these variables, we use a bijection between Minkowski space $\mathbb{R}^{1,3}$ and the space of Hermitian $2 \times 2$ matrices. A Minkowski vector $p=\left(p_{\mu}\right)$ is represented by the matrix

$$
\left(p_{\alpha \dot{\beta}}\right)=\left(\begin{array}{cc}
p_{0}+p_{3} & p_{1}-i p_{2} \\
p_{1}+i p_{2} & p_{0}-p_{3}
\end{array}\right),
$$

where the indices take the values $\alpha, \dot{\beta}=1,2$. Using the Minkowski inner product $p \cdot q=$ $p_{0} q_{0}-\vec{p} \cdot \vec{q}$, one verifies that $\operatorname{det}\left(p_{\alpha \dot{\beta}}\right)=p^{2}$. Hence, for the null momenta of the scattering process, the corresponding matrix is at most of rank 1 and can thus be factorized as

$$
p_{\alpha \dot{\beta}}=\lambda_{\alpha} \tilde{\lambda}_{\dot{\beta}}
$$

with two spinors $\lambda=\left(\lambda_{\alpha}\right), \tilde{\lambda}=\left(\tilde{\lambda}_{\dot{\beta}}\right) \in \mathbb{C}^{2}$. To turn this into a Hermitian matrix, we can without loss of generality restrict to spinors satisfying the reality condition

$$
\tilde{\lambda}= \pm \bar{\lambda}
$$

The sign here determines the sign of the energy as $\pm 2 p_{0}=\left|\lambda_{1}\right|^{2}+\left|\lambda_{2}\right|^{2}$. In the field of scattering amplitudes, one often works with complexified momenta, i.e. independent spinors $\lambda$ and $\tilde{\lambda}$ that do not obey the reality condition (2.3). This condition will be of utmost importance for our work. Let us briefly motivate why we prefer spinor helicity variables to twistors or momentum twistors is this article. First, it is easy to work with real momenta by imposing (2.3). What is more, the variables $\lambda, \tilde{\lambda}$ together with the fermions $\tilde{\eta}$ are associated with the superconformal algebra $\mathfrak{p s u}(2,2 \mid 4)$. They straightforwardly generalize to certain oscillator representations of the superalgebra $\mathfrak{u}(p, q \mid m)$, which will play an important role in section 5 . 
After setting up the formalism, we can discuss actual amplitudes $\mathcal{A}_{N, K}$. In fact, expressions for all $\mathcal{A}_{N, K}$ are known [10]. They involve the spinors in terms of the Lorentz invariant angle and square brackets,

$$
\langle i j\rangle=\lambda_{1}^{i} \lambda_{2}^{j}-\lambda_{2}^{i} \lambda_{1}^{j}, \quad[i j]=-\tilde{\lambda}_{1}^{i} \tilde{\lambda}_{2}^{j}+\tilde{\lambda}_{2}^{i} \tilde{\lambda}_{1}^{j} .
$$

Making use of (2.3), these two brackets are related by

$$
[i j]=-\operatorname{sgn}\left(p_{0}^{i}\right) \operatorname{sgn}\left(p_{0}^{j}\right) \overline{\langle i j\rangle} .
$$

Moreover, the amplitudes contain generalized Mandelstam variables that can be expanded in terms of the brackets,

$$
s_{i j \cdots k}=\left(p^{i}+p^{j}+\ldots+p^{k}\right)^{2}=\sum_{\substack{u<v \\ \in\{i, j, \ldots, k\}}}\langle u v\rangle[v u] .
$$

Here we represent only the maximally helicity violating (MHV) amplitudes explicitly, i.e. $K=2$. They are given by the unexpectedly simple Parke-Taylor formula [11], or rather its supersymmetric extension [12],

$$
\mathcal{A}_{N, 2}=\frac{\delta^{4}(P) \delta^{0 \mid 8}(Q)}{\langle 12\rangle\langle 23\rangle \cdots\langle N-1 N\rangle\langle N 1\rangle},
$$

which holds for $N \geq 4$. Momentum and supermomentum conservation are implemented by

$$
\begin{aligned}
\delta^{4}(P) & =\delta\left(P_{11}\right) \delta\left(P_{22}\right) \delta\left(\operatorname{Re} P_{21}\right) \delta\left(\operatorname{Im} P_{21}\right) & \text { with } \quad P_{\alpha \dot{\beta}} & =\sum_{i=1}^{N} \lambda_{\alpha}^{i} \tilde{\lambda}_{\dot{\beta}}^{i}, \\
\delta^{0 \mid 8}(Q) & =\prod_{\alpha=1}^{2} \prod_{\dot{a}=1}^{4} Q_{\alpha \dot{a}} & \text { with } \quad Q_{\alpha \dot{a}} & =\sum_{i=1}^{N} \lambda_{\alpha}^{i} \tilde{\eta}_{\dot{a}}^{i} .
\end{aligned}
$$

We stress that for momentum conservation to hold, both signs in (2.3) are needed, i.e. there have to be particles with positive and negative energy. In a setting with complexified momenta, (2.7) also yields a three-particle amplitude $\mathcal{A}_{3,2}$. However, for spinors obeying (2.3), this ceases to exist for purely kinematic reasons, as explained e.g. in [2].

\subsection{Yangian symmetry}

The tree-level amplitudes $\mathcal{A}_{N, K}$ are invariant under an infinite-dimensional symmetry algebra, the Yangian of the superconformal algebra $\mathfrak{p s u}(2,2 \mid 4)$. Let us start our discussion a bit more general and consider the Yangian of the Lie superalgebra $\mathfrak{g l}(n \mid m)[13,14]$. An introduction to Yangians is provided e.g. in the recent lectures [15]. Arguably, the most elegant way to define these algebras is in the context of the quantum inverse scattering method (QISM), which explores the consequences of the Yang-Baxter equation to provide a toolbox for the study of integrable models, see the authoritative review [16]. In this language the generators of the Yangian are packaged into the entries of a spin chain monodromy matrix. A Yang-Baxter equation obeyed by this monodromy yields the commutation relations of the generators. 
In what follows, we specify this monodromy matrix $M(u)$ in detail to derive explicit formulas for the Yangian generators. It is built out of Lax operators

$$
L_{i}\left(u-v_{i}\right)=1+\left(u-v_{i}\right)^{-1} \sum_{\mathcal{A}, \mathcal{B}} E_{\mathcal{A B}} J_{\mathcal{B} \mathcal{A}}^{i}(-1)^{|\mathcal{B}|}=\square-\left.\cdots\right|_{i} ^{---}
$$

where $u$ and $v_{i}$ are complex parameters referred to as spectral parameter and inhomogeneity, respectively. The Lax operator acts on the tensor product $\square \otimes \mathscr{V}_{i}$ of $\mathfrak{g l}(n \mid m)$ representations. The generators $E_{\mathcal{A B}}$ of the defining representation $\square=\mathbb{C}^{n \mid m}$ are supermatrices satisfying in particular $E_{\mathcal{A B}} E_{\mathcal{C D}}=\delta_{\mathcal{B C}} E_{\mathcal{A D}}$. The generators of the representation $\mathscr{V}_{i}$ are denoted $J_{\mathcal{A B}}^{i}$. Both sets of generators obey the $\mathfrak{g l}(n \mid m)$ algebra

$$
\left[J_{\mathcal{A B}}, J_{\mathcal{C D}}\right\}=\delta_{\mathcal{C B}} J_{\mathcal{A D}}-(-1)^{(|\mathcal{A}|+|\mathcal{B}|)(|\mathcal{C}|+|\mathcal{D}|)} \delta_{\mathcal{A D}} J_{\mathcal{C B}}
$$

with superindices such as $\mathcal{A}=1, \ldots, n+m$ whose degree $|\mathcal{A}|=0,1$ depends on the grading. Out of these Lax operators, we construct the monodromy matrix of an inhomogeneous spin chain with $N$ sites,

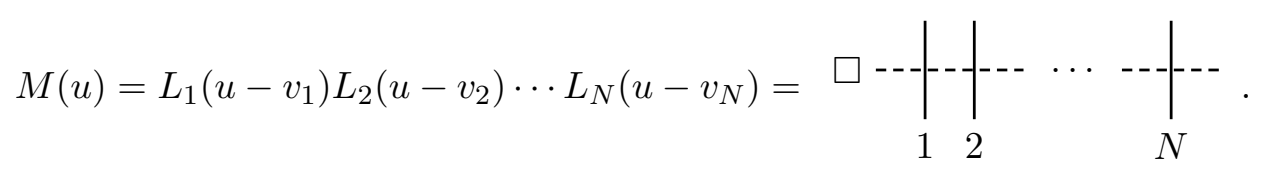

The product of Lax operators here is considered to be a matrix product in the space $\square$ and a tensor product in the spaces $\mathscr{V}_{i}$. The generators $M_{\mathcal{A B}}^{(l)}$ with $l=1,2,3, \ldots$ of the Yangian are obtained by expanding ${ }^{1}$ the elements of the monodromy matrix in the spectral parameter $u$,

$$
\begin{aligned}
M(u) & =\sum_{\mathcal{A}, \mathcal{B}} E_{\mathcal{A B}} M_{\mathcal{A B}}(u)(-1)^{|\mathcal{B}|} \\
M_{\mathcal{A B}}(u) & =\delta_{\mathcal{A B}}(-1)^{|\mathcal{B}|}+u^{-1} M_{\mathcal{A B}}^{(1)}+u^{-2} M_{\mathcal{A B}}^{(2)}+\ldots
\end{aligned}
$$

With the Lax operators in (2.9), these generators acting in $\mathscr{V}_{1} \otimes \cdots \otimes \mathscr{V}_{N}$ read

$$
M_{\mathcal{A B}}^{(1)}=\sum_{i=1}^{N} J_{\mathcal{B} \mathcal{A}}^{i}, \quad M_{\mathcal{A B}}^{(2)}=\sum_{i=1}^{N} v_{i} J_{\mathcal{B} \mathcal{A}}^{i}+\sum_{\substack{i, j=1 \\ i<j}}^{N} \sum_{\mathcal{C}}(-1)^{|\mathcal{C}|} J_{\mathcal{B C}}^{j} J_{\mathcal{C} \mathcal{A}}^{i}, \quad \ldots
$$

We will encounter this form of the Yangian generators in sections 3 and 5 . In the context of $\mathcal{N}=4$ SYM scattering amplitudes, one usually works with slightly different looking generators $M_{\mathcal{A B}}^{[l]}$. These are obtained from another expansion of the monodromy matrix,

$$
M(u)=1+u^{-1} M^{(1)}+u^{-2} M^{(2)}+\ldots=\exp \left(u^{-1} M^{[1]}+u^{-2} M^{[2]}+\ldots\right) .
$$

We will provide explicit formulas for the generators $M_{\mathcal{A B}}^{[l]}$ momentarily, see (2.20) below.

\footnotetext{
${ }^{1}$ The powers of $u$ in this expansion motivate our labeling of the generators by $l=1,2,3, \ldots$ despite the frequent use of the "levels" $l-1=0,1,2, \ldots$ for this purpose.
} 
Of central importance are Yangian invariants. These are states $|\Psi\rangle \in \mathscr{V}_{1} \otimes \cdots \otimes \mathscr{V}_{N}$ that are annihilated by all Yangian generators,

$$
M_{\mathcal{A B}}^{(l)}|\Psi\rangle=0
$$

for all $l=1,2,3, \ldots$. Due to the commutation relations of the generators, see e.g. [1], it is actually sufficient to verify this condition only for the first two sets of generators with $l=1,2$. The Yangian invariance condition (2.15) can be expressed equivalently in terms of the generators $M_{\mathcal{A} \mathcal{B}}^{[l]}$. In addition, it takes a natural form when written employing the spin chain monodromy $(2.11)[17,18]$,

$$
M(u)|\Psi\rangle=1|\Psi\rangle
$$

where the identity operator on the right-hand side acts on $\square=\mathbb{C}^{n \mid m}$. We may represent this equation graphically as
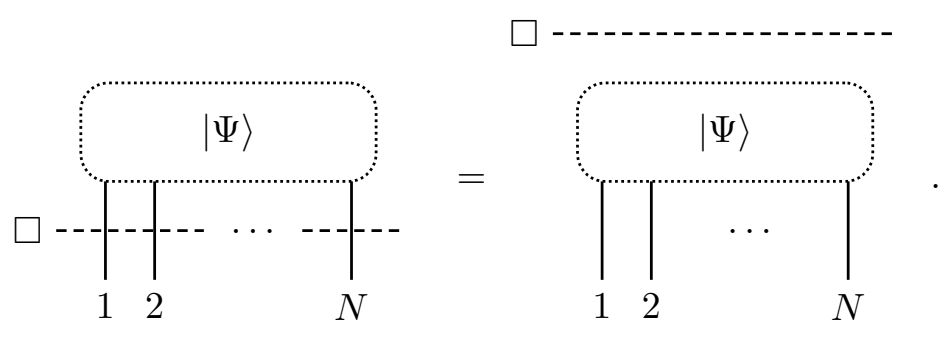

This brings Yangian invariants inside the realm of the QISM. Hence, it potentially allows to construct them using the associated tools, such as the algebraic Bethe ansatz [17].

After this detour, we return to the tree-level amplitudes $\mathcal{A}_{N, K}$ of planar $\mathcal{N}=4 \mathrm{SYM}$. Their Yangian invariance was discovered in [19] by combining the well-known invariance under the superconformal algebra $\mathfrak{p s u}(2,2 \mid 4)$ with a rather unexpected occurrence of a second copy of $\mathfrak{p s u}(2,2 \mid 4)$ termed dual superconformal symmetry. Reviews on the Yangian symmetry of amplitudes and on its relevance for other observables of planar $\mathcal{N}=4 \mathrm{SYM}$ are provided in $[15,20,21]$. Instead of following the historic route, we apply the formalism introduced in the preceding paragraphs to arrive at Yangian generators that annihilate the amplitudes $\mathcal{A}_{N, K}$.

A function annihilated by the generators of $\mathfrak{p s u}(2,2 \mid 4)$ is also annihilated by any complex linear combination thereof and hence by the complexified algebra $\mathfrak{p s l}\left(\mathbb{C}^{4 \mid 4}\right) \equiv \mathfrak{p s l}(4 \mid 4)$. Generators $\mathfrak{J}_{\mathcal{A B}}$ of $\mathfrak{g l}(4 \mid 4) \supset \mathfrak{p s l}(4 \mid 4)$ are easily realized in terms of spinor helicity variables. Arranged into a supermatrix they read

$$
\left(\mathfrak{J}_{\mathcal{A B}}\right)=\left(\begin{array}{c:c:c}
\lambda_{\alpha} \partial_{\lambda_{\beta}} & \lambda_{\alpha} \tilde{\lambda}_{\dot{\beta}} & \lambda_{\alpha} \tilde{\eta}_{\dot{b}} \\
\hdashline-\partial_{\tilde{\lambda}_{\dot{\alpha}}} \partial_{\lambda_{\beta}} & -\partial_{\tilde{\lambda}_{\dot{\alpha}}} \tilde{\lambda}_{\dot{\beta}} & -\partial_{\tilde{\lambda}_{\dot{\alpha}}} \tilde{\eta}_{\dot{b}} \\
\hdashline \partial_{\tilde{\eta}_{\dot{a}}} \partial_{\lambda_{\beta}} & \partial_{\tilde{\eta}_{\dot{a}}} \tilde{\lambda}_{\dot{\beta}} & \partial_{\tilde{\eta}_{\dot{a}}} \tilde{\eta}_{\dot{b}}
\end{array}\right) .
$$

Here we split the superindices such as $\mathcal{A}=1, \ldots, 8$ into bosonic indices $\alpha, \dot{\alpha}=1,2$ and a fermionic index $\dot{a}=1,2,3,4$. In order to restrict to the algebra $\mathfrak{p s l}(4 \mid 4)$, we have to impose 
$\mathfrak{C}=\operatorname{tr}\left(\mathfrak{J}_{\mathcal{A B}}\right)=\sum_{\mathcal{A}} \mathfrak{J}_{\mathcal{A} \mathcal{A}}=0$ and $\mathfrak{B}=\operatorname{str}\left(\mathfrak{J}_{\mathcal{A B}}\right)=\sum_{\mathcal{A}}(-1)^{|\mathcal{A}|} \mathfrak{J}_{\mathcal{A} \mathcal{A}}=0$. Generators of $\mathfrak{s l}(4 \mid 4)$ are obtained from (2.18) by defining

$$
\mathfrak{J}_{\mathcal{A B}}^{\prime}=\mathfrak{J}_{\mathcal{A B}}-\frac{1}{8}(-1)^{|A|} \delta_{\mathcal{A B}} \mathfrak{B}
$$

They satisfy $\mathfrak{C}^{\prime}=\mathfrak{C}$ and $\mathfrak{B}^{\prime}=0$. Let us consider a spin chain monodromy matrix $M(u)$ as in (2.11) with generators $J_{\mathcal{A B}}^{i}=\mathfrak{J}_{\mathcal{A B}}^{i}$ of the form (2.18) in the Lax operators. Expanding this monodromy as in (2.14) yields the Yangian generators

$$
M_{\mathcal{A B}}^{[1]}=\sum_{i=1}^{N} \mathfrak{J}_{\mathcal{B A}}^{i}, \quad M_{\mathcal{A B}}^{[2]}=\frac{1}{2} \sum_{\substack{i, j=1 \\ i<j}}^{N} \sum_{\mathcal{C}}(-1)^{|\mathcal{C}|}\left(\tilde{J}_{\mathcal{B C}}^{j} \mathfrak{J}_{\mathcal{C} \mathcal{A}}^{i}-\mathfrak{J}_{\mathcal{B C}}^{i} \mathfrak{J}_{\mathcal{C} \mathcal{A}}^{j}\right)+\sum_{i=1}^{N} \hat{v}_{i} \mathfrak{J}_{\mathcal{B} \mathcal{A}}^{i}
$$

To obtain these expressions, we used the form of the generators in (2.18), assumed $\sum_{i=1}^{N} \mathfrak{C}^{i}=$ 0 , and introduced $\hat{v}_{i}=v_{i}-\frac{c_{i}}{2}+\frac{1}{2}$ with $c_{i}$ being the eigenvalue of $\mathfrak{C}^{i}$. For the amplitudes, these eigenvalues are closely related to the superhelicities of the particles, and they have to vanish, $c_{i}=0$. In order to act with $(2.20)$ on the amplitudes, we also have to set $\hat{v}_{i}=0$. Then the amplitudes are annihilated by the Yangian generators after replacing the $\mathfrak{g l}(4 \mid 4)$ generators $\mathfrak{J}_{\mathcal{A B}}^{i}$ in $(2.20)$ by the $\mathfrak{s l}(4 \mid 4)$ generators from (2.19), which we indicate by apostrophes,

$$
M_{\mathcal{A B}}^{\prime[1]} \mathcal{A}_{N, K}=0, \quad M_{\mathcal{A B}}^{\prime[2]} \mathcal{A}_{N, K}=0
$$

This is the form of the Yangian invariance condition typically found in the amplitudes literature, see e.g. [19]. The first equation in (2.21) is the ordinary action of a Lie superalgebra on a tensor product and corresponds to superconformal invariance.

We remark that a careful analysis, taking into account the reality conditions (2.3) of the spinor variables, reveals of a breaking of the Yangian invariance (2.21), and even the superconformal invariance alone, at certain singularities of the amplitudes [22-24]. Because this issue does not occur for generic particle momenta, it is often neglected in the discussion of tree-level amplitudes.

\subsection{Graßmannian integral and deformations}

In this section, we discuss a very compact formulation of the amplitudes $\mathcal{A}_{N, K}$ in terms of certain multi-dimensional contour integrals called Graßmannian integrals [25, 26], see also the extensive treatise ${ }^{2}[28]$. This approach particularly suites our interests because it allows for an easy investigation of symmetries. While the superconformal symmetry is manifest in this formulation, also the Yangian symmetry can be verified [29, 30].

Before defining the Graßmannian integral, we have to discuss some bare essentials of Graßmannian manifolds. The complex Graßmannian $\operatorname{Gr}(K, N)$ is defined as the space of all $K$-dimensional linear subspaces of $\mathbb{C}^{N}$. "Homogeneous" coordinates on this space are provided by the complex entries of a $K \times N$ matrix $C$. Selecting a basis within a given

\footnotetext{
${ }^{2}$ This book project grew out of the influential preprint [27].
} 
subspace does not alter the point in the Graßmannian. Consequently, a generic point in $\operatorname{Gr}(K, N)$ can be described by the "gauge fixed" matrix

$$
C=\left(1_{K} \mathcal{C}\right) \quad \text { with } \quad \mathcal{C}=\left(\begin{array}{ccc}
C_{1 K+1} & \cdots & C_{1 N} \\
\vdots & & \vdots \\
C_{K K+1} & \cdots & C_{K N}
\end{array}\right)
$$

where $1_{K}$ denotes the $K \times K$ unit matrix. In what follows, we will also encounter the $(N-K) \times N$ matrix $C^{\perp}=\left(-\mathcal{C}^{t} 1_{N-K}\right)$ obeying $C\left(C^{\perp}\right)^{t}=0$. It is an element of $\operatorname{Gr}(N-$ $K, N)$. These ingredients are sufficient to present the Graßmannian integral formulation of amplitudes [25],

$$
\mathcal{A}_{N, K}=\int \mathrm{d}^{K(N-K)} \mathcal{C} \frac{\delta_{*}^{2(N-K) \mid 0}\left(C^{\perp} \boldsymbol{\lambda}\right) \delta_{*}^{2 K \mid 0}(C \tilde{\boldsymbol{\lambda}}) \delta^{0 \mid 4 K}(C \tilde{\boldsymbol{\eta}})}{(1, \ldots, K) \cdots(N, \ldots, K-1)}
$$

with the holomorphic form $\mathrm{d}^{K(N-K)} \mathcal{C}=\bigwedge_{k, l} \mathrm{~d} C_{k l}$. Here $(i, \ldots, i+K-1)$ signifies the minor of the matrix $C$ constructed from the consecutive columns $i, \ldots, i+K-1$. These are counted modulo $N$ such that they stay within the range $1, \ldots, N$. The external data is encoded in the $N \times 2$ matrices $\boldsymbol{\lambda}=\left(\lambda_{\alpha}^{i}\right)$ and $\tilde{\boldsymbol{\lambda}}=\left(\tilde{\lambda}_{\dot{\alpha}}^{i}\right)$ as well as the $N \times 4$ matrix $\tilde{\boldsymbol{\eta}}=\left(\tilde{\eta}_{\dot{a}}^{i}\right)$. The symbol $\delta_{*}$ denotes a formal bosonic delta function whose argument may be complex. It can be understood as a calculation rule to set the argument to zero and omit an integration. To evaluate the Graßmannian integral (2.23), one first uses the formal bosonic delta functions to reduce the number of integration variables. Then one specifies a contour for the remaining variables, which has to be closed to ensure Yangian invariance. The resulting integral can be evaluated by means of a multi-dimensional generalization of Cauchy's residue theorem, the so-called "global residue theorem", see the discussion in [25]. Suitable contours are known for all amplitudes $\mathcal{A}_{N, K}$. They can be specified in a geometric fashion [31], generalizing partial results in [32, 33].

Let us mention a significant open problem of the Graßmannian integral approach in the form presented in (2.23). In case of the physical Minkowski signature $(1,3)$, the spinors obey the reality conditions (2.3). Hence, the spinors contained in $\tilde{\boldsymbol{\lambda}}$ depend on those in $\boldsymbol{\lambda}$. This is neglected in the standard way of evaluating the integral, which we just sketched. The issue is commonly evaded by working in split signature $(2,2)$ or in a complexified momentum space, where $\boldsymbol{\lambda}$ and $\tilde{\boldsymbol{\lambda}}$ are treated as independent real or complex variables, respectively.

In discussing the Yangian invariance of the amplitudes $\mathcal{A}_{N, K}$ around (2.21), we noted that we had to set $c_{i}=0$ and $\hat{v}_{i}=0$ in the Yangian generators (2.20). This naturally leads to the question whether there exist deformed amplitudes $\mathcal{A}_{N, K}^{\text {(def) }}$ for which these parameters do not vanish. It was first raised in [34,35], one motivation being that complex deformation parameters might serve as integrability-based regulators of loop amplitudes. In addition, the deformations should be crucial to properly understand the integrable structure of amplitudes and to eventually put this structure to use for their efficient construction, even at all-loop level. The study of deformed amplitudes continued in [36-38]. It resulted in a 
deformed Graßmannian integral [39, 40]

$$
\mathcal{A}_{N, K}^{(\text {def })}=\int_{?} \mathrm{~d}^{K(N-K)} \mathcal{C} \frac{\delta_{*}^{2(N-K) \mid 0}\left(C^{\perp} \boldsymbol{\lambda}\right) \delta_{*}^{2 K \mid 0}(C \tilde{\boldsymbol{\lambda}}) \delta^{0 \mid 4 K}(C \tilde{\boldsymbol{\eta}})}{(1, \ldots, K)^{1+\hat{v}_{K}^{-}-\hat{v}_{1}^{+}} \cdots(N, \ldots, K-1)^{1+\hat{v}_{K-1}^{-}-\hat{v}_{N}^{+}}} .
$$

Here the exponents are defined by [36]

$$
\hat{v}_{i}^{ \pm}=\hat{v}_{i} \pm \frac{c_{i}}{2} .
$$

They have to satisfy

$$
\hat{v}_{i+K}^{-}=\hat{v}_{i}^{+}
$$

for $i=1, \ldots, N$, where we count modulo $N$. This condition ensures the Yangian invariance (2.21) of the integral (2.24), provided a closed integration contour. Furthermore, starting from the Yangian invariance condition (2.16) involving a spin chain monodromy, (2.24) can even be derived using tools rooted in the QISM [39]. These tools were introduced in $[18,41]$ and studied more systematically in [37, 42]. However, this method uses somewhat formal integral operators and does not yield a suitable contour for the resulting deformed Graßmannian integral (2.24). Deformations of the MHV amplitudes (2.7) can be obtained from (2.24) without specifying a contour because all integration variables are fixed by the bosonic delta functions,

$$
\mathcal{A}_{N, 2}^{(\mathrm{def})}=\frac{\delta^{4}(P) \delta^{0 \mid 8}(Q)}{\langle 12\rangle^{1+\hat{v}_{2}^{-}-\hat{v}_{1}^{+}} \cdots\langle N 1\rangle^{1+\hat{v}_{1}^{-}-\hat{v}_{N}^{+}}} .
$$

This brings us to the main challenge of understanding the deformed amplitudes $\mathcal{A}_{N, K}^{\text {(def) }}$. It is not known how to evaluate the integral (2.24) beyond the MHV case. Because of the complex exponents of the minors in the denominator, Cauchy's residue theorem and its multi-dimensional generalization do not apply any longer. The exponents lead to branch cuts and the resulting multi-valuedness of the integrand makes it very difficult to find a closed integration contour, which is necessary for Yangian invariance. We will address this problem in section 3. It turns out that the choice of the integration contour is tightly interrelated with using the proper Minkowski reality conditions (2.3) for the spinors in $\boldsymbol{\lambda}$ and $\tilde{\boldsymbol{\lambda}}$.

Finally, let us mention that the Yangian invariance condition for $\mathcal{A}_{4,2}^{(\mathrm{def})}$ can be shown to be equivalent to a Yang-Baxter equation. This suggests interpreting $\mathcal{A}_{4,2}^{\text {(def) }}$ as an R-matrix, where one of its deformation parameters is the spectral parameter of this R-matrix [34, 35]. In this interpretation, the undeformed amplitude $\mathcal{A}_{4,2}$ corresponds to the R-matrix evaluated at a special point of the spectral parameter. Interestingly, this interpretation appears to be at odds with [43], where $\mathcal{A}_{4,2}$ was related to the one-loop dilatation operator of the planar $\mathcal{N}=4 \mathrm{SYM}$ spectral problem. This operator is not an R-matrix itself but can be constructed from one. We believe that this conceptual difference deserves further attention and will revisit it in section 6 . 


\section{Unitary Graßmannian integral}

Our aim in this section is to construct a refined version of the deformed Graßmannian integral (2.24) that respects the reality conditions (2.3) of the spinor helicity variables in Minkowski signature. We will start with what seems to be the most involved step, finding a suitable integration contour. Then we can formulate the sought after refined Graßmannian formula. In particular, we discuss the crucial single-valuedness of its integrand. Finally, we define Yangian generators that annihilate our integral formula and specify the occurring representations.

\subsection{Reality conditions and unitary contour}

First, we have to introduce our setting. From now on, we restrict ourselves to the case $N=2 K$, which corresponds to helicity conserving amplitudes. It has the technical advantage that the integration variable $\mathcal{C}$ defined in (2.22) is a complex $K \times K$ square matrix. Instead of focusing on $\mathfrak{p s u}(2,2 \mid 4)$ Yangian invariants relevant for the amplitudes $\mathcal{A}_{2 K, K}$, we immediately generalize to $\mathfrak{u}(p, p \mid m)$. This generalization can be done almost effortlessly, and it will help us to gain important insights ${ }^{3}$ into the structure of our integrals later. It implies replacing the bosonic variables $\lambda, \tilde{\lambda} \in \mathbb{C}^{2}$ defined in $(2.2)$ by $\lambda, \tilde{\lambda} \in \mathbb{C}^{p}$. Even though in general these variables are not associated with four-dimensional Minkowski momenta anymore, it is often helpful to continue using this terminology. To be able to perform concrete calculations, we have to impose the reality conditions (2.3) on the variables $\lambda^{i}, \tilde{\lambda}^{i} \in \mathbb{C}^{p}$. We choose negative energies for the first $K$ momenta and positive energies for the latter $K$. This completely determines the $2 K \times p$ matrix $\tilde{\boldsymbol{\lambda}}$ containing all $\tilde{\lambda}^{i}$ in terms of the matrix $\boldsymbol{\lambda}$ containing the $\lambda^{i}$,

$$
\tilde{\lambda}=\left(\begin{array}{c}
\tilde{\lambda}^{-} \\
\hdashline \tilde{\lambda}^{+}
\end{array}\right)=\left(\begin{array}{c}
-\bar{\lambda}^{-} \\
\cdots \ldots \ldots \ldots \\
\bar{\lambda}^{+}
\end{array}\right), \quad \lambda=\left(\begin{array}{c}
\lambda^{-} \\
\cdots \ldots \ldots \\
\lambda^{+}
\end{array}\right) .
$$

The $K \times p$ blocks of $\boldsymbol{\lambda}$ are $\boldsymbol{\lambda}^{-}=\left(\lambda_{\alpha}^{k}\right)$ and $\boldsymbol{\lambda}^{+}=\left(\lambda_{\alpha}^{l}\right)$, whose row indices run over $k=$ $1, \ldots, K$ and $l=K+1, \ldots, 2 K$, respectively. We will stick to this setup throughout this article.

Now we are in a position to discuss the integration contour. A characteristic feature of the Graßmannian integral (2.24), that we want to keep for our refined version, is the linear relations among the spinors in $\boldsymbol{\lambda}$ imposed by bosonic delta functions,

$$
0=C^{\perp} \boldsymbol{\lambda}=-\mathcal{C}^{t} \boldsymbol{\lambda}^{-}+\boldsymbol{\lambda}^{+} .
$$

Furthermore, we want to impose momentum conservation, recall (2.8),

$$
P_{\alpha \dot{\beta}}=\sum_{i=1}^{N} \lambda_{\alpha}^{i} \tilde{\lambda}_{\dot{\beta}}^{i}=0 \quad \Leftrightarrow \quad \lambda^{t} \tilde{\boldsymbol{\lambda}}=0 .
$$

\footnotetext{
${ }^{3}$ In particular, it will be instructive to study the simpler algebra $\mathfrak{u}(1,1)$ in section 4.3 below. This may remind the reader of the Amplituhedron [44]. Its definition involves a parameter $m$, and it yields physical amplitudes for $m=4$. At times, it is investigated for the mathematically more accessible case $m=2$, which corresponds to $\mathfrak{u}(1,1 \mid 2)$ in our language.
} 
Here the total momentum is encoded in the Hermitian $p \times p$ matrix $P=\left(P_{\alpha \dot{\beta}}\right)$. For our purpose, it is more suitable to work with its expression in terms of the matrices $\boldsymbol{\lambda}$ and $\tilde{\boldsymbol{\lambda}}$. With the help of (3.1) and (3.2), we obtain

$$
0=\lambda^{t} \tilde{\boldsymbol{\lambda}}=\left(\tilde{\boldsymbol{\lambda}}^{-}\right)^{\dagger}\left(\mathcal{C} \mathcal{C}^{\dagger}-1_{K}\right) \tilde{\boldsymbol{\lambda}}^{-}
$$

This is most naturally satisfied by demanding $\mathcal{C} \in \mathrm{U}(K)$. It strikingly suggests that the contour of our refined version of the Graßmannian integral (2.24) should be a unitary group manifold. See also appendix B for an independent argument in favor of this contour.

Clearly, in order to cover all amplitudes $\mathcal{A}_{N, K}$, it would be necessary to extend the reasoning presented in this section to the case $N \neq 2 K$ and, in addition, to allow for arbitrary choices of the energy signs. We comment on both of these aspects separately. The unitary group manifold seems to generalize to a so-called Stiefel manifold ${ }^{4}$ for $N \neq$ $2 K$. Starting with the linear constraint $C^{\perp} \boldsymbol{\lambda}=0$ as in (3.2), we arrive again at (3.4), where now $\mathcal{C}$ from $(2.22)$ is a non-square $K \times(N-K)$ matrix. Assuming $N>2 K$, we can demand $\mathcal{C C}^{\dagger}=1_{K}$, which defines said manifold. However, this condition has no solution in case of $N<2 K$. Here we resort to the other constraint $C \tilde{\boldsymbol{\lambda}}=0$ from the Graßmannian integral (2.24), which leads to the Stiefel manifold $\mathcal{C}^{\dagger} \mathcal{C}=1_{N-K}$. Note that the two constraints become equivalent for $N=2 K$ due to the unitarity of $\mathcal{C}$. Eventually, one should also generalize the distribution of the energy signs. As long as there are $K$ particles with negative and $N-K$ with positive energy, we can align the gauge fixing of the matrix $C$ in (2.22) with the distribution of signs in (3.1). In this way, we obtain a Stiefel manifold for the matrix block $\mathcal{C}$ once again. Additional complications surface for different numbers of positive and negative energy particles. This is a bit puzzling because the energy signs enter the final formula [10] for the amplitude $\mathcal{A}_{N, K}$ only mildly via the reality conditions $(2.5)$ of the spinor brackets $[i j]$. We leave the extensions discussed in this paragraph for future work as already our specific setting will give rise to rich structures.

\subsection{Graßmannian integral}

Here we implement the insight on the choice of the contour. In doing so, we generalize the superalgebra slightly further by allowing for different gradings ${ }^{5}$ of the fermions, which we indicate by the notation $\mathfrak{u}(p, p \mid m=r+s)$. We define a unitary Graßmannian integral computing Yangian invariants for this algebra by

$$
\Psi_{N=2 K, K}=\int_{\mathrm{U}(K)}[\mathrm{d} \mathcal{C}] \mathscr{F}(\mathcal{C}) \delta_{\mathbb{C}}^{p K \mid 0}\left(C^{\perp} \boldsymbol{\lambda}\right) \delta^{0 \mid r K}\left(C^{\perp} \boldsymbol{\eta}\right) \delta^{0 \mid s K}(C \tilde{\boldsymbol{\eta}}),
$$

\footnotetext{
${ }^{4}$ We will encounter this manifold, together with the appropriate Haar measure, for a different purpose in appendix B.

${ }^{5}$ This might seem unnecessarily tedious. In fact, we will exclusively use the grading $\mathfrak{u}(2,2 \mid 0+4)$ to relate our results to amplitudes in this article. However, for the spectral problem of planar $\mathcal{N}=4 \mathrm{SYM}$, the grading $\mathfrak{u}(2,2 \mid 2+2)$ appears to be more natural at one loop [45,46] and is the key to all-loop results such as [47]. We will discuss the prospect of extending our formalism to the results of the latter reference in section 6.1 .
} 
where the Graßmannian matrices $C$ and $C^{\perp}$ are defined around (2.22). They contain the matrix block $\mathcal{C}$ that we impose be to unitary here. We denote the Haar measure on the unitary group $\mathrm{U}(K)$ by $[\mathrm{d} \mathcal{C}]$. The constraint (3.2) is imposed in the integrand using complex delta functions, which are defined by $\delta_{\mathbb{C}}(z)=\delta(\operatorname{Re} z) \delta(\operatorname{Im} z)$ for $z \in \mathbb{C}$. The $2 K \times p$ matrix $\boldsymbol{\lambda}$ contains the bosonic variables $\lambda^{i} \in \mathbb{C}^{p}$ associated with the sites $i=1, \ldots, 2 K$, as explained around (3.1). For the representations we are working with, each site is also associated with $r$ - and $s$-dimensional fermionic variables $\eta^{i}$ and $\tilde{\eta}^{i}$, respectively. They are packaged into the $2 K \times r$ matrix $\boldsymbol{\eta}=\left(\eta_{a}^{i}\right)$ and the $2 K \times s$ matrix $\tilde{\boldsymbol{\eta}}=\left(\tilde{\eta}_{\dot{a}}^{i}\right)$. A characteristic part of the integrand is the function

$$
\mathscr{F}(\mathcal{C})^{-1}=(\operatorname{det} \mathcal{C})^{m-q-K} \prod_{i=1}^{2 K}(i, \ldots, i+K-1)^{1+v_{i+K-1}^{-}-v_{i}^{+}},
$$

which contains the minors $(i, \ldots, i+K-1)$ of the matrix $C$. For its use in (3.5), we have to identify $q=p$. In section 5 below, we will need $\mathscr{F}(\mathcal{C})$ without this identification. The exponents of the minors are given by

$$
v_{i}^{ \pm}=v_{i}^{\prime} \pm \frac{c_{i}}{2}, \quad v_{i}^{\prime}=v_{i}-\frac{c_{i}}{2}+ \begin{cases}n-m-1 & \text { for } \quad i=1, \ldots, K, \\ 0 & \text { for } \quad i=K+1, \ldots, 2 K,\end{cases}
$$

where $n=2 p$ and $m=r+s$. The case distinction originates from the choice of energy signs for the sites in (3.1). The parameters $v_{i} \in \mathbb{C}$ are inhomogeneities of a spin chain monodromy matrix (2.11) whose sites carry $\mathfrak{u}(p, p \mid r+s)$ representations labeled by $c_{i} \in \mathbb{Z}$, see the discussion in section 3.4 below. To enable the Yangian invariance of $\Psi_{2 K, K}$ in (3.5), we have to demand

$$
v_{i+K}^{-}=v_{i}^{+}
$$

for $i=1, \ldots, 2 K$. We denote the Yangian invariants by $\Psi_{2 K, K}$ instead of $\mathcal{A}_{2 K, K}^{\text {(def) }}$ because we still have to establish their relation to the amplitudes $\mathcal{A}_{2 K, K}$. This will be achieved for sample invariants in section 4 , and a further perspective on their relation will be added in section 6.2 .

Let us compare the unitary integral (3.5) with the original deformed Graßmannian integral (2.24). First, there are no delta functions explicitly constraining $\tilde{\boldsymbol{\lambda}}$ in (3.5). This would be superfluous because the delta functions containing $\boldsymbol{\lambda}$ together with the reality conditions in (3.1) yield the constraint $C \tilde{\boldsymbol{\lambda}}=0$. Next, the Haar measure $[\mathrm{d} \mathcal{C}]$ in $(3.5)$ can easily be obtained from the holomorphic form in (2.24),

$$
[\mathrm{d} \mathcal{C}] \propto \frac{\mathrm{d}^{K^{2}} \mathcal{C}}{(\operatorname{det} \mathcal{C})^{K}}
$$

for $\mathcal{C} \in \mathrm{U}(K)$. The proportionality constant is fixed by demanding $\int_{\mathrm{U}(K)}[\mathrm{d} \mathcal{C}]=1$. This form of the Haar measure is suitable for showing its left- und right-invariance, i.e. invariance under the transformation $\mathcal{C} \mapsto \mathcal{V C W}$ with constant matrices $\mathcal{V}, \mathcal{W} \in \mathrm{U}(K)$. What is more, the function $\mathscr{F}(\mathcal{C})^{-1}$ from (3.6) essentially reduces to the product of minors in (2.24). The 
additional factors of $\operatorname{det} \mathcal{C}$ can be attributed to the algebra $\mathfrak{u}(p, p \mid m)$ generalizing $\mathfrak{p} \mathfrak{s u}(2,2 \mid 4)$, the arguments of the bosonic delta functions, and the Haar measure. An important change is the restriction from complex representation labels $c_{i}$ in (2.24) to integer ones in (3.5). It is required for the single-valuedness of $\mathscr{F}(\mathcal{C})$ addressed in the next section.

\subsection{Single-valued integrand}

The main obstruction to finding a closed contour for the original deformed Graßmannian integral (2.24), needed to show its Yangian invariance, is the intricate branch cut structure of its integrand caused by the complex exponents of the minors. Even though the unitary group $\mathrm{U}(K)$ is compact, it could still fail to yield a closed contour for our integral (3.5), if the integrand $\mathscr{F}(\mathcal{C})$ from $(3.6)$ was multi-valued. Until now, this integrand is still formal because we have not specified its analytic structure yet. In what follows, we will do this implicitly by manipulating it into a form that is explicitly single-valued.

We start by expressing the minors of the $K \times 2 K$ matrix $C$ defined in (2.22) in terms of those of the $K \times K$ matrix $\mathcal{C}$,

$$
(i, \ldots, i+K-1)=(-1)^{(K-i+1)(i-1)} \begin{cases}{[1, \ldots, i-1]} & \text { for } \quad i=1, \ldots, K \\ {[i-K, \ldots, K]} & \text { for } \quad i=K+1, \ldots, 2 K\end{cases}
$$

In this formula, the principal minor of $\mathcal{C}$ built from the rows and columns $i$ to $j$ is denoted $[i, \ldots, j]$, e.g. [ $]=1,[1]=C_{1 K+1}$, and $[1, \ldots, K]=\operatorname{det} \mathcal{C}$. Furthermore, using the unitarity of $\mathcal{C}$ we obtain

$$
[i+1, \ldots, K]=\overline{[1, \ldots, i]} \operatorname{det} \mathcal{C},
$$

see e.g. [48]. This useful identity can be proven using a block decomposition of $\mathcal{C}$. It is helpful to translate the constraints on $v_{i}^{ \pm}$in (3.8) into constraints on the parameters $v_{i}, c_{i}$,

$$
v_{K+i}=v_{i}+n-m-1-c_{i}, \quad c_{K+i}=-c_{i},
$$

for $i=1, \ldots K$. Using the relations obtained here and disregarding the analytic structure temporarily by combining products of minors with common complex exponents, we rewrite (3.6), up to a constant sign factor, as

$$
\mathscr{F}(\mathcal{C})^{-1}=(\operatorname{det} \mathcal{C})^{m-q+c_{K}} \prod_{i=1}^{K-1}|[1, \ldots, i]|^{2\left(1+v_{i}-v_{i+1}\right)}\left[\overline{[1, \ldots, i]}^{c_{i+1}-c_{i}}\right.
$$

This function is manifestly single-valued because only non-negative numbers are exponentiated to non-integer powers. Here we see the paramount importance of integer representation labels $c_{i}$. Henceforth, we will use the single-valued integrand $\mathscr{F}(\mathcal{C})$ defined in $(3.13)$ instead of formal expression (3.6). Consequently, the unitary integration contour in (3.5) is closed, as is required to demonstrate Yangian invariance. 


\subsection{Symmetry generators and Yangian invariance}

We still have to specify the Yangian generators annihilating the invariants $\Psi_{2 K, K}$ defined by the unitary Graßmannian integral (3.5). For this purpose, we need to introduce some basics of two classes of $\mathfrak{u}(p, p \mid r+s)$ representations. In the special case of $\mathfrak{s u}(2,2)$, these are wellknown representations of the conformal algebra [49, 50] and the two classes correspond to positive and negative energies. In general, they are equivalent to certain oscillator representations $[51,52]$, which we will encounter in section 5 .

For the first class of representations, we consider generators $\mathfrak{J}_{\mathcal{A B}}$ of the $\mathfrak{g l}(n \mid m)$ superalgebra (2.10) built from the bosonic variables $\lambda_{\alpha} \in \mathbb{C}$ and the fermions $\eta_{a}$ and $\tilde{\eta}_{\dot{a}}$ with the index ranges $\alpha=1, \ldots, p, a=1, \ldots, r$, and $\dot{a}=1, \ldots, s$. They are given by the supermatrix

$$
\left(\mathfrak{J}_{\mathcal{A B}}\right)=\left(\begin{array}{c:c:c:c}
\lambda_{\alpha} \partial_{\lambda_{\beta}} & \lambda_{\alpha} \partial_{\eta_{b}} & \lambda_{\alpha} \bar{\lambda}_{\beta} & \lambda_{\alpha} \tilde{\eta}_{\dot{b}} \\
\hdashline \eta_{a} \partial_{\lambda_{\beta}} & \eta_{a} \partial_{\eta_{b}} & \eta_{a} \bar{\lambda}_{\beta} & \eta_{a} \tilde{\eta}_{\dot{b}} \\
\hdashline-\partial_{\bar{\lambda}_{\alpha}} \partial_{\lambda_{\beta}} & -\partial_{\bar{\lambda}_{\alpha}} \partial_{\eta_{b}} & -\partial_{\bar{\lambda}_{\alpha}} \bar{\lambda}_{\beta} & -\partial_{\bar{\lambda}_{\alpha}} \tilde{\eta}_{\dot{b}} \\
\hdashline \partial_{\tilde{\eta}_{\dot{a}}} \partial_{\lambda_{\beta}} & \partial_{\tilde{\eta}_{\dot{a}}} \partial_{\eta_{b}} & \partial_{\tilde{\eta}_{\dot{a}}} \bar{\lambda}_{\beta} & \partial_{\tilde{\eta}_{\dot{a}}} \tilde{\eta}_{\dot{b}}
\end{array}\right)
$$

with superindices $\mathcal{A}, \mathcal{B}=1, \ldots, 2 p+r+s=n+m$. Let $\mathscr{D}_{c}$ denote the space of those functions of the bosonic and fermionic variables on which the central element

$$
\mathfrak{C}=\operatorname{tr}\left(\mathfrak{J}_{\mathcal{A B}}\right)=\sum_{\alpha=1}^{p}\left(\lambda_{\alpha} \partial_{\lambda_{\alpha}}-\bar{\lambda}_{\alpha} \partial_{\bar{\lambda}_{\alpha}}\right)+\sum_{a=1}^{r} \eta_{a} \partial_{\eta_{a}}-\sum_{\dot{a}=1}^{s} \tilde{\eta}_{\dot{a}} \partial_{\tilde{\eta}_{\dot{a}}}-p+s
$$

acts by multiplication with the eigenvalue $c$. We define conjugates of the variables by

$$
\lambda_{\alpha}^{\dagger}=\bar{\lambda}_{\alpha}, \quad \partial_{\lambda_{\alpha}}^{\dagger}=-\partial_{\bar{\lambda}_{\alpha}}, \quad \eta_{a}^{\dagger}=\partial_{\eta_{a}}, \quad \tilde{\eta}_{\dot{a}}^{\dagger}=\partial_{\tilde{\eta}_{\dot{a}}} .
$$

At this point, we refrain from stating the inner product leading to this choice, which would also be required for a less formal definition of the space $\mathscr{D}_{c}$. Some details will be filled in below in sections 5.3 and 5.4. With (3.16), $\mathscr{D}_{c}$ for $c \in \mathbb{Z}$ carries a unitary representation of $\mathfrak{u}(p, p \mid r+s)$. For the algebra $\mathfrak{u}(2,2 \mid 0+4)$, the supermatrix of generators (3.14) reduces to (2.18) with the reality condition $\tilde{\lambda}=+\bar{\lambda}$ from (2.3). Hence, in slight abuse of terminology, we refer to the $\mathscr{D}_{c}$ also for the algebra $\mathfrak{u}(p, p \mid r+s)$ as positive energy representation. The second class of representations is obtained from the $\mathfrak{g l}(n \mid m)$ generators

$$
\overline{\mathfrak{J}}_{\mathcal{A B}}=\left.\mathfrak{J}_{\mathcal{A B}}\right|_{(\lambda, \bar{\lambda}) \mapsto(\lambda,-\bar{\lambda})}+\delta_{\mathcal{A B}}(-1)^{|\mathcal{A}|},
$$

where $\mathfrak{J}_{\mathcal{A B}}$ are the generators in (3.14). We have

$$
\overline{\mathfrak{C}}=\operatorname{tr}\left(\overline{\mathfrak{J}}_{\mathcal{A B}}\right)=\sum_{\alpha=1}^{p}\left(\lambda_{\alpha} \partial_{\lambda_{\alpha}}-\bar{\lambda}_{\alpha} \partial_{\bar{\lambda}_{\alpha}}\right)+\sum_{a=1}^{r} \eta_{a} \partial_{\eta_{a}}-\sum_{\dot{a}=1}^{s} \tilde{\eta}_{\dot{a}} \partial_{\tilde{\eta}_{\dot{a}}}+p-r .
$$

The space of functions on which this central element acts by multiplication with $c \in \mathbb{Z}$ is denoted $\overline{\mathscr{D}}_{c}$ and forms a unitary representation of $\mathfrak{u}(p, p \mid r+s)$. We refer to $\overline{\mathscr{D}}_{c}$ as negative energy representation. 
Let us remark that for $\mathfrak{u}(2,2 \mid 0+4)$ the $\overline{\mathfrak{J}}_{\mathcal{A B}}$ differ from the generators in (2.18) with $\tilde{\lambda}=-\bar{\lambda}$ by the second term in (3.17). This term arises naturally in section 5.4 , where we will revisit the representations introduced here. Practically, its inclusion has the advantage that we do not have to change from $\mathfrak{g l}(n \mid m)$ to $\mathfrak{s l}(n \mid m)$ generators, as it is usually the case for the $\mathcal{N}=4 \mathrm{SYM}$ amplitudes $\mathcal{A}_{N, K}$, cf. (2.19) and (2.21). On a different note, the expressions for the central elements $\mathfrak{C}$ and $\overline{\mathfrak{C}}$ in (3.15) and (3.18), respectively, agree for $2 p=r+s$. In particular, this is the case for $\mathfrak{u}(2,2 \mid 0+4)$, where these generators essentially measure the superhelicities of particles described by the amplitudes $\mathcal{A}_{N, K}$.

Finally, given the two classes of representations, we are able to construct a monodromy matrix $M(u)$ with $N=2 K$ sites, as introduced in (2.11), that is associated with $\Psi_{2 K, K}$ defined by the unitary Graßmannian integral (3.5). With our choice of energy signs in (3.1), the first $K$ sites carry negative energy representations $\overline{\mathscr{D}}_{c_{i}}$ and the latter $K$ sites carry positive energy representations $\mathscr{D}_{c_{i}}$. Hence the generators entering the Lax operators (2.9) of the monodromy are

$$
J_{\mathcal{A B}}^{i}= \begin{cases}\overline{\mathfrak{J}}_{\mathcal{A B}}^{i} & \text { for } \quad i=1, \ldots, K \\ \mathfrak{J}_{\mathcal{A B}}^{i} & \text { for } \quad i=K+1, \ldots, 2 K\end{cases}
$$

which can be found in (3.17) and (3.14), respectively. The inhomogeneities $v_{i}$ and representations labels $c_{i}$ of the monodromy have to obey (3.8), recall also the equivalent equation (3.12). Then $\Psi_{2 K, K}$ defined in (3.5) satisfies the Yangian invariance condition (2.15) and thus also (2.16) in the QISM language. We do not provide a direct proof of this key statement here. Instead, we refer the reader to section 5 , where we translate $\Psi_{2 K, K}$ into a different basis in which the Yangian invariance has been proven.

\section{Sample invariants and amplitudes}

Having defined Yangian invariants by the unitary Graßmannian integral (3.5), we evaluate this integral here for several examples. Our primary focus are sample invariants $\Psi_{2 K, K}$ for the algebra $\mathfrak{u}(2,2 \mid 4)$ whose relation to the $\mathcal{N}=4 \mathrm{SYM}$ amplitudes $\mathcal{A}_{2 K, K}$ we explore. The first step in the evaluation of (3.5) can be performed on general grounds, even for $\mathfrak{u}(p, p \mid r+s)$. It is possible to reduce ${ }^{6}$ the $\mathrm{U}(K)$ integral to a $\mathrm{U}(K-p)$ integral provided $K \geq p$. Technically, this reduction is based on QR decompositions of the $K \times p$ blocks $\lambda^{ \pm}$ making up the kinematic data in $\boldsymbol{\lambda}$, cf. (3.1). The unitary factors of these decompositions can be absorbed into the integration variable $\mathcal{C} \in \mathrm{U}(K)$ using the left- and right-invariance of the Haar measure. The bosonic delta functions in (3.5) then reduce the new integration variable to a $\mathrm{U}(K-p)$ matrix. On a different note, our evaluation of the integral (3.5) in the present section addresses only terms with maximal kinematic support, i.e. the those proportional to the momentum conserving delta function (2.8). We neglect additional terms that can appear for special kinematic configurations, see section 6.2 below.

\footnotetext{
${ }^{6}$ This is somewhat similar to the transition [53] from the original $G r(K, N)$ Graßmannian integral (2.23) for $\mathcal{A}_{N, K}$ in spinor helicity variables to that in terms of momentum twistors involving the Graßmannian $\operatorname{Gr}(K-2, N)$.
} 


\subsection{Four particles for $\mathfrak{u}(2,2 \mid 4)$}

As a first example, we evaluate the integral (3.5) to obtain the invariant $\Psi_{4,2}$ for the algebra $\mathfrak{u}(2,2 \mid 0+4)$. Our naming of the fermionic variables suggests that this grading of the algebra is required to make contact with the amplitude $\mathcal{A}_{4,2}$ from (2.7). The bosonic delta functions in (3.5) fix the integration variable completely,

$$
\mathcal{C}=\frac{1}{\langle 12\rangle}\left(\begin{array}{ll}
\langle 32\rangle & \langle 42\rangle \\
\langle 13\rangle & \langle 14\rangle
\end{array}\right)
$$

This is a unitary matrix because of the reality conditions of the spinors in (3.1) and momentum conservation (3.3). We obtain from it the Yangian invariant

$$
\Psi_{4,2}=\frac{\delta^{4 \mid 0}(P) \delta^{0 \mid 8}(Q)}{\langle 12\rangle\langle 23\rangle\langle 34\rangle\langle 41\rangle}\left(\frac{\langle 14\rangle}{\langle 34\rangle}\right)^{c_{1}}\left(\frac{\langle 12\rangle}{\langle 14\rangle}\right)^{c_{2}}\left(\frac{\langle 34\rangle \overline{\langle 34\rangle}}{\langle 14\rangle \overline{\langle 14\rangle}}\right)^{v_{1}-v_{2}},
$$

where we dropped a numerical prefactor, as we will also do in the following examples. The delta functions implementing momentum and supermomentum conservation are defined in (2.8). We chose to display the result using complex conjugates of angle brackets $\overline{\langle i j\rangle}$ instead of square brackets $[i j]$ to highlight the analytic structure, recall the relation between these brackets from (2.5). $\Psi_{4,2}$ is a single-valued function of the spinors $\lambda_{\alpha}^{i}$ because the factor raised to the complex power $v_{1}-v_{2}$ is non-negative and $c_{1}, c_{2} \in \mathbb{Z}$. Moreover, $\Psi_{4,2}$ basically agrees with the deformed amplitude $\mathcal{A}_{4,2}^{\text {(def) }}$ from (2.27), which was first obtained in [34]. This is leaving aside the crucial restriction to integer representation labels $c_{1}, c_{2}$ and a slight, inessential difference in the parameterization of the complex deformation parameters. $\Psi_{4,2}$ reduces to the amplitude $\mathcal{A}_{4,2}$ from (2.7) for $c_{1}=c_{2}=0$ and $v_{1}=v_{2}$. Let us remark that evaluating the Graßmannian integral (3.5) for $\Psi_{4,2}$ in case of the bosonic algebra $\mathfrak{u}(2,2)$ with these deformation parameters yields the four-particle tree-level MHV gluon amplitude with the split helicity configuration $(+1,+1,-1,-1)$.

\subsection{Six particles for $\mathfrak{u}(2,2 \mid 4)$}

Let us move on to six particles. It was argued in [36] that a deformation of the amplitude $\mathcal{A}_{6,3}$ cannot be constructed by deforming the individual residues contributing to it. Does this imply that there is no such deformation? The authors of [39] put forward the idea of deforming the entire integral from which the residues are extracted. This resulted in the Graßmannian integral (2.24) for the deformed amplitude $\mathcal{A}_{6,3}^{(\text {def })}$. However, as discussed in section 2.3, a suitable contour for this integral has been missing so far. Here we evaluate our Graßmannian integral formula (3.5) with the unitary contour for the $\mathfrak{u}(2,2 \mid 0+4)$ Yangian invariant $\Psi_{6,3}$. In particular, we show how it reduces to $\mathcal{A}_{6,3}$ in the undeformed limit.

First, the bosonic delta functions in (3.5) determine the integration variable $\mathcal{C} \in \mathrm{U}(3)$ up to a phase $\mathcal{U} \in \mathrm{U}(1)$,

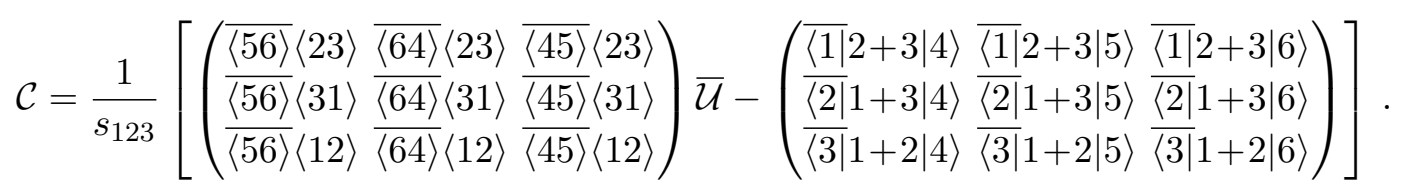


The unitarity of this matrix can be verified using the reality conditions in (3.1) and momentum conservation (3.3). This reduces the $\mathrm{U}(3)$ integral (3.5) to the $\mathrm{U}(1)$ integral

$$
\begin{aligned}
\Psi_{6,3}= & \frac{\delta^{4 \mid 0}(P) \delta^{0 \mid 8}(Q)}{s_{123}^{5}} \int_{\mathrm{U}(1)}[\mathrm{d} \mathcal{U}] \frac{\delta^{0 \mid 4}(\mathrm{a} \mathcal{U}+\mathrm{b})}{\mathcal{U}^{2-c_{3}}|\mathrm{~A}-\mathcal{U B}|^{2\left(1+v_{1}-v_{2}\right)}(\mathrm{A}-\mathcal{U B})^{c_{2}-c_{1}}} \\
& \cdot \frac{1}{|\mathcal{U} \mathrm{C}-\mathrm{D}|^{2\left(1+v_{2}-v_{3}\right)}(\mathcal{U} \mathrm{C}-\mathrm{D})^{c_{3}-c_{2}}}
\end{aligned}
$$

with the kinematic data encoded in the variables

$$
\begin{array}{ll}
\mathrm{A}=\frac{\langle 1| 2+3 \overline{|4\rangle}}{s_{123}}, \quad \mathrm{~B}=\frac{\langle 56\rangle \overline{\langle 23\rangle}}{s_{123}}, \quad \mathrm{C}=\frac{\overline{\langle 3|} 1+2|6\rangle}{s_{123}}, \quad \mathrm{D}=\frac{\overline{\langle 45\rangle}\langle 12\rangle}{s_{123}}, \\
\mathrm{a}=\overline{\langle 23\rangle} \tilde{\eta}^{1}+\overline{\langle 31\rangle} \tilde{\eta}^{2}+\overline{\langle 12\rangle} \tilde{\eta}^{3}, & \mathrm{~b}=\overline{\langle 56\rangle} \tilde{\eta}^{4}+\overline{\langle 64\rangle} \tilde{\eta}^{5}+\overline{\langle 45\rangle} \tilde{\eta}^{6} .
\end{array}
$$

Here $\tilde{\eta}^{i}=\left(\tilde{\eta}_{\dot{a}}^{i}\right)$ are four-dimensional fermionic variables and the momentum and supermomentum conserving delta functions are defined in (2.8). The one-dimensional integral (4.4) is our final expression for the fully deformed Yangian invariant $\Psi_{6,3}$. It would be desirable to express it in terms of known special functions.

Next, to make contact with the amplitude $\mathcal{A}_{6,3}$, we study the undeformed limit of (4.4), i.e. we set $c_{1}=c_{2}=c_{3}=0$ and $v_{1}=v_{2}=v_{3}$. This yields the complex contour integral

$$
\Psi_{6,3}=\frac{\delta^{4 \mid 0}(P) \delta^{0 \mid 8}(Q)}{s_{123}^{5}} \frac{1}{2 \pi i \overline{\mathrm{ABC}} \overline{\mathrm{D}}} \oint \mathrm{d} \mathcal{U} \frac{\delta^{0 \mid 4}(\mathrm{a} \mathcal{U}+\mathrm{b})}{\mathcal{U}\left(\mathcal{U}-\frac{\mathrm{A}}{\overline{\mathrm{B}}}\right)\left(\mathcal{U}-\frac{\overline{\bar{B}}}{\overline{\mathrm{A}}}\right)\left(\mathcal{U}-\frac{\mathrm{D}}{\mathrm{C}}\right)\left(\mathcal{U}-\frac{\overline{\overline{\mathrm{C}}}}{\overline{\mathrm{D}}}\right)},
$$

where we wrote the Haar measure as $[\mathrm{d} \mathcal{U}]=\frac{1}{2 \pi i} \frac{\mathrm{d} \mathcal{U}}{\mathcal{U}}$, cf. (3.9), and thereby parameterized $\mathrm{U}(1)$ as the counterclockwise unit circle in the complex $\mathcal{U}$-plane. This integral can be computed by means of Cauchy's residue theorem. The pole at $\mathcal{U}=0$ obviously lies inside of the contour. In addition, there are two pairs of poles, whose positions depend on the kinematic data. If the pole at $\mathcal{U}=\frac{\mathrm{A}}{\bar{B}}$ is inside of the contour, the one at $\mathcal{U}=\frac{\overline{\mathrm{B}}}{\overline{\mathrm{A}}}$ is outside, and vice versa. The same holds true for the pair at $\mathcal{U}=\frac{\mathrm{D}}{\mathrm{C}}, \frac{\overline{\mathrm{C}}}{\overline{\mathrm{D}}}$. An illustration of this behavior is presented in figure 1 . As a result, there are always three residues contributing to the integral (4.6). Notice that here we neglect the possibility of poles moving onto the contour because this is only possible for special kinematics. The selection of contributing residues is controlled by the signs of the variables

$$
1-\left|\frac{\mathrm{A}}{\mathrm{B}}\right|^{2}=\frac{s_{234} s_{123}}{s_{23} s_{56}} \propto s_{234}, \quad 1-\left|\frac{\mathrm{C}}{\mathrm{D}}\right|^{2}=\frac{s_{345} s_{123}}{s_{12} s_{45}} \propto s_{345} .
$$

We expressed them in terms of the Mandelstam variables from (2.6). Our selection of energy signs in (3.1) implies that $s_{123}$ and the $s_{i j}$ appearing in (4.7) are non-negative. Thus we dropped these factors in the last step.

In order to be able to compare the undeformed invariant (4.6) with the amplitude $\mathcal{A}_{6,3}$, we translate the residues into the R-invariants [54]

$$
\mathcal{R}^{r ; s t}=\frac{\langle s s-1\rangle\langle t t-1\rangle \delta^{0 \mid 4}\left(\left\langle r\left|x^{r s} x^{s t}\right| \theta^{t r}\right\rangle+\left\langle r\left|x^{r t} x^{t s}\right| \theta^{s r}\right\rangle\right)}{\left(x^{s t}\right)^{2}\left\langle r\left|x^{r s} x^{s t}\right| t\right\rangle\left\langle r\left|x^{r s} x^{s t}\right| t-1\right\rangle\left\langle r\left|x^{r t} x^{t s}\right| s\right\rangle\left\langle r\left|x^{r t} x^{t s}\right| s-1\right\rangle} .
$$




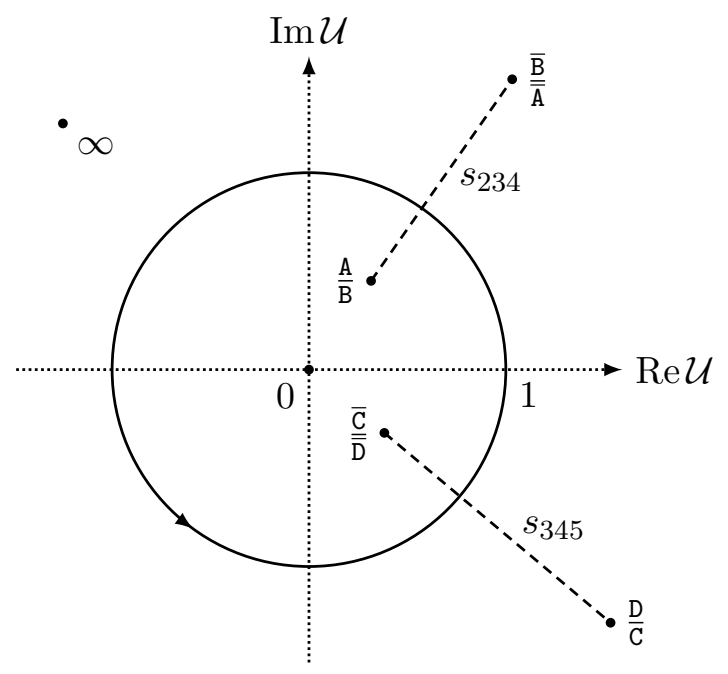

Figure 1. Sample configuration of the poles of the integrand in (4.6) for the undeformed $\mathfrak{u}(2,2 \mid 4)$ Yangian invariant $\Psi_{6,3}$. Dashed lines connect pairs of poles. For each pair, exactly one pole is inside of the contour. The signs of the Mandelstam variables $s_{234}$ and $s_{345}$ determine which one.

These are expressed in terms of the dual variables $x^{i}$ and $\theta^{i}$. They are defined by the relations $\lambda_{\alpha}^{i} \tilde{\lambda}_{\dot{\beta}}^{i}=x_{\alpha \dot{\beta}}^{i}-x_{\alpha \dot{\beta}}^{i+1}$ and $\lambda_{\alpha}^{i} \tilde{\eta}_{\dot{a}}^{i}=\theta_{\alpha \dot{a}}^{i}-\theta_{\alpha \dot{a}}^{i+1}$ with the identification $N+1 \equiv 1$ for the particle indices, where here $N=6$. Furthermore, we used the abbreviations $x^{i j}=x^{i}-x^{j}$ and $\theta^{i j}=\theta^{i}-\theta^{j}$. The dual superconformal symmetry mentioned in section 2.2 acts naturally on the variables $x^{i}$ and $\theta^{i}$. Employing the R-invariants from (4.8), the undeformed integral (4.6) evaluates to

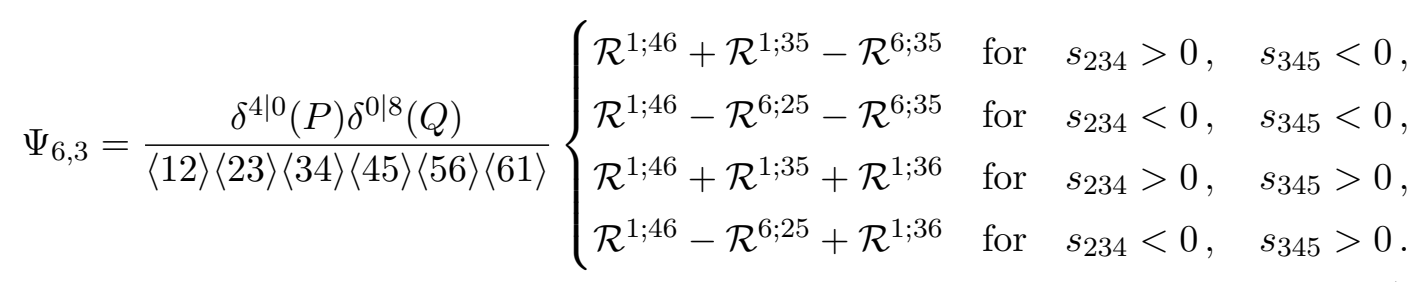

Here the four different kinematic regions arise from the residue positions in (4.7). Each $\mathcal{R}^{r ; s t}$ in (4.9) corresponds to one residue. For example the term with $\mathcal{R}^{1 ; 46}$ is associated with the residue of the integrand in $(4.6)$ at $\mathcal{U}=0$ because it appears in all regions. The other associations can be deduced similarly. In the kinematic region $s_{234}, s_{345}>0$, the undeformed invariant $\Psi_{6,3}$ in (4.9) agrees with the amplitude $\mathcal{A}_{6,3}[54,55]$. Hence the $\mathrm{U}(3)$ contour we started out with in the Graßmannian integral (3.5) automatically selects the desired residues in this region. Curiously, it also implies the emergence of three other kinematic regions, in which (4.9) does not match the amplitude. This makes us speculate that the Yangian symmetry of the known expression for the amplitude $\mathcal{A}_{6,3}$ could be broken in a subtle way because it misses the region structure. We will strengthen this point in the following section. There we compute the unitary Graßmannian integral (3.5) for a simpler Yangian invariant, that serves as a toy model for $\Psi_{6,3}$ of $\mathfrak{u}(2,2 \mid 4)$ considered here. 
In that case, we are able to show the necessity of the emerging kinematic regions from an integrability perspective.

We remark that the four kinematic regions in (4.9) appeared also in the study of hexagonal light-like Wilson loops in [56]. The two fractions of Mandelstam variables in (4.7), which define the regions, are two of the three independent dual conformal cross ratios defined in equation (1) of that reference. The authors investigate the possible values of these cross ratios. Our choice of energy signs in (3.1) corresponds to the case considered in their equation (40). In this case, the allowed values form four distinct regions in the cross ratio space $\mathbb{R}^{3}$, which match our regions in (4.9) and are nicely visualized in their figure 1.

\subsection{Toy model and implications: four particles for $\mathfrak{u}(1,1)$}

Here we evaluate the unitary Graßmannian integral (3.5) for the $\mathfrak{u}(1,1)$ Yangian invariant $\Psi_{4,2}$. This is the simplest invariant for which different kinematic regions emerge in the undeformed limit. In this sense, it is a toy model for the example studied in the previous section. We begin the evaluation by reducing the U(2) integral in (3.5) to a U(1) integral with the help of the bosonic delta functions,

$$
\Psi_{4,2}=\frac{\delta(P)}{\left|\lambda^{1}\right|^{2}+\left|\lambda^{2}\right|^{2}} \int_{\mathrm{U}(1)}[\mathrm{d} \mathcal{U}] \frac{1}{\mathcal{U}^{1-c_{2}}|\mathrm{~A}-\mathcal{U B}|^{2\left(1+v_{1}-v_{2}\right)}(\mathrm{A}-\mathcal{U B})^{c_{2}-c_{1}}}
$$

Here $P=-\left|\lambda^{1}\right|^{2}-\left|\lambda^{2}\right|^{2}+\left|\lambda^{3}\right|^{2}+\left|\lambda^{4}\right|^{2}$, and we introduced

$$
\mathrm{A}=\frac{\lambda^{1} \bar{\lambda}^{3}}{\left|\lambda^{1}\right|^{2}+\left|\lambda^{2}\right|^{2}}, \quad \mathrm{~B}=\frac{-\lambda^{4} \bar{\lambda}^{2}}{\left|\lambda^{1}\right|^{2}+\left|\lambda^{2}\right|^{2}} .
$$

Notice that we write $\lambda_{1}^{i} \equiv \lambda^{i}$ because these variables have only one component in case of the algebra $\mathfrak{u}(1,1)$. The integral (4.10) can be expressed in terms of a Gauß hypergeometric function and even more specifically an associated Legendre function, see [1]. We move on to study the undeformed limit of (4.10) by setting $c_{1}=c_{2}=0$ and $v_{1}=v_{2}$. This yields

$$
\Psi_{4,2}=-\frac{\delta(P)}{\left|\lambda^{1}\right|^{2}+\left|\lambda^{2}\right|^{2}} \frac{1}{2 \pi i \overline{\mathrm{AB}}} \oint \mathrm{d} \mathcal{U} \frac{1}{\mathcal{U}\left(\mathcal{U}-\frac{\mathrm{A}}{\overline{\mathrm{B}}}\right)\left(\mathcal{U}-\frac{\overline{\mathrm{B}}}{\overline{\mathrm{A}}}\right)},
$$

where, as after (4.6), we reformulated the Haar measure on $\mathrm{U}(1)$ in terms of a contour integral in the complex variable $\mathcal{U}$. Thus we integrate counterclockwise along the unit circle. Just like for the example in the previous section, we compute this integral using the residue theorem. The pole at $\mathcal{U}=0$ contributes to the integral irrespective of the kinematics. In contrast to the previous section, there is now only one pair poles at $\mathcal{U}=\frac{\mathrm{A}}{\mathrm{B}}, \overline{\overline{\mathrm{B}}}$ that depends on the kinematics, cf. figure 2. For generic kinematics, precisely one of these poles lies inside of the contour. Which one is determined by the sign of the variable

$$
1-\left|\frac{\mathrm{A}}{\mathrm{B}}\right|^{2} \propto\left|\lambda^{2}\right|^{2}-\left|\lambda^{3}\right|^{2}
$$




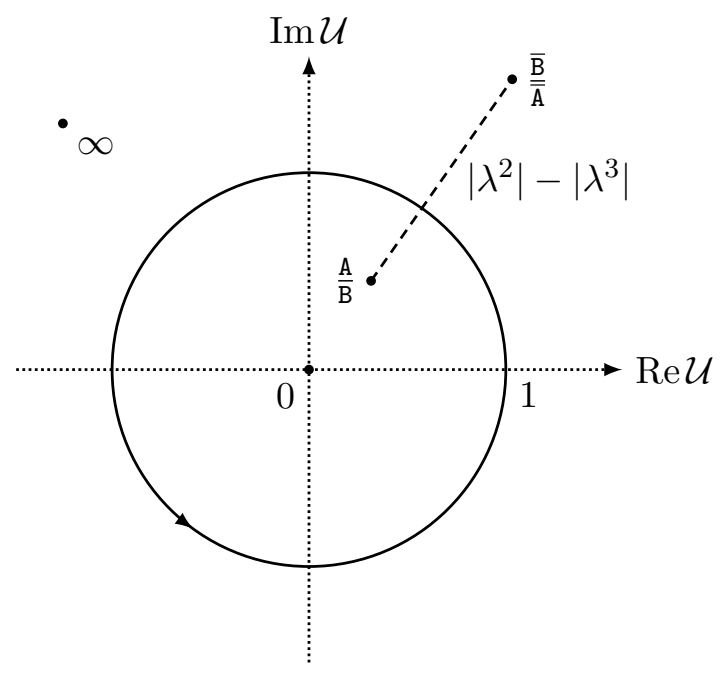

Figure 2. Sample pole configuration of the integrand in (4.12) for the undeformed $\mathfrak{u}(1,1)$ Yangian invariant $\Psi_{4,2}$. A pair of poles is connected by a dashed line. The sign of the variable $\left|\lambda^{2}\right|-\left|\lambda^{3}\right|$ controls which of the poles lies inside of the contour.

where we used $P=0$ and dropped a non-negative factor. Summing the two residues contributing to (4.12) gives the undeformed invariant

$$
\Psi_{4,2}=\frac{\delta(P)}{\left|\lambda^{2}\right|^{2}-\left|\lambda^{3}\right|^{2}}\left\{\begin{array}{rll}
\frac{\lambda^{4} \overline{\lambda^{2}}}{\lambda^{1} \lambda^{3}} & \text { for } & \left|\lambda^{2}\right|<\left|\lambda^{3}\right|, \\
-\frac{\overline{\lambda^{1}} \lambda^{3}}{\bar{\lambda}^{4} \lambda^{2}} & \text { for } & \left|\lambda^{2}\right|>\left|\lambda^{3}\right|
\end{array}\right.
$$

with two kinematic regions.

Let us now show that these two regions are required by integrability. Our argument is based on a parity transformation $\mathcal{P}$ of the Graßmannian integral (3.5) discussed in appendix A. In case of the toy model under investigation here, this transformation boils down to exchanging the spinors $\lambda^{1} \stackrel{\mathcal{P}}{\leftrightarrow} \lambda^{2}, \lambda^{3} \stackrel{\mathcal{P}}{\leftrightarrow} \lambda^{4}$. It is a symmetry of the $\mathfrak{u}(1,1)$ Yangian invariant $\Psi_{4,2}$ in (4.10) for general deformation parameters $v_{1}, v_{2}$ and equal representation labels $c_{1}=c_{2}$. In section 6 below, we will see that this invariant can be interpreted as an R-matrix. Its symmetry under $\mathcal{P}$ translates into the well-known parity invariance of this R-matrix. With this background knowledge, we study the action of $\mathcal{P}$ on the undeformed $\Psi_{4,2}$ in (4.14). We find that it exchanges the two kinematic regions. Let us discuss what would happen if we used the expression of either region in (4.14) and declared it to be valid for all kinematics. The resulting function would still satisfy the Yangian invariance condition (2.15), which takes the form of a set of differential equations in $\lambda^{i}$, for generic values of $\lambda^{i}$. However, it would clearly violate parity symmetry. Therefore the two kinematic regions in (4.14) are required for the proper Yangian invariant, which is related to the R-matrix.

Can we exploit the parity transformation $\mathcal{P}$ from appendix A also to show the necessity of the four kinematic regions of the undeformed $\mathfrak{u}(2,2 \mid 0+4)$ Yangian invariant $\Psi_{6,3}$ in (4.9) for integrability? This is not possible because the region $s_{234}, s_{345}>0$, in which the 
invariant agrees with the amplitude $\mathcal{A}_{6,3}$, is by itself invariant under $\mathcal{P}$. The transformation $\mathcal{P}$ is part of a known dihedral symmetry of the amplitude $\mathcal{A}_{6,3}$, cf. [57]. In fact, it is the only non-trivial element of that group which is compatible with our choice of energy signs in (3.1). It would be interesting to look for a bigger discrete symmetry group of (4.9) that connects all four regions and is needed for an integrability-based reason. Because $\Psi_{6,3}$ can be interpreted as a product of three R-matrices, see once again section 6 below, we suspect that such a bigger symmetry group could involve parity transformations of the individual R-matrices and the Yang-Baxter equation satisfied by the product. So far this equation has been verified for the amplitude $\mathcal{A}_{6,3}$ merely on the level of the original Graßmannian integral or equivalently for on-shell diagrams [27, 35], disregarding in both cases the integration contour. The existence of such a discrete symmetry group of (4.9) would signal a subtle breakdown of integrability in the formula $[54,55]$ for the amplitude $\mathcal{A}_{6,3}$.

It is also conceivable that the region structure of the undeformed Yangian invariant $\Psi_{6,3}$ in (4.9) is a sign of a broken conformal symmetry of the amplitude $\mathcal{A}_{6,3}$. This is currently under investigation [58]. Both quantities are certainly invariant under infinitesimal transformations of the conformal algebra $\mathfrak{s u}(2,2) \subset \mathfrak{u}(2,2 \mid 0+4)$ for generic values of the spinors $\lambda^{i}$. However, to the best of our knowledge, the invariance of $\mathcal{A}_{6,3}$ under finite transformations has not been proven. The representations of $\mathfrak{s u}(2,2)$ in terms of spinors $\lambda^{i}$ from section 3.4 can be exponentiated to ones of the group $\mathrm{SU}(2,2)[59,60]$. In particular, some group elements act quite non-trivially as integral transformations. We remark that kinematic regions somewhat reminiscent of those in (4.9) were introduced for NMHV amplitudes in split signature $(2,2)$ to obtain amplitudes with satisfactory superconformal properties in twistor space [61].

\subsection{Eight particles for $\mathfrak{u}(2,2 \mid 4)$}

In our investigation of the four- and six-particle $\mathfrak{u}(2,2 \mid 0+4)$ Yangian invariants in the previous sections, we could extract the respective amplitudes, at least in one kinematic region, by setting all deformation parameters to zero. Obviously, this raises the question if, in general, the amplitude $\mathcal{A}_{2 K, K}$ can be obtained from the Yangian invariant $\Psi_{2 K, K}$ in this simple way. Therefore we continue our studies in this section by computing $\Psi_{8,4}$. Vexingly, we will find that the simple procedure does not hold up for extracting the amplitude $\mathcal{A}_{8,4}$ from it.

To begin with, we reduce the U(4) Graßmannian integral formula (3.5) for the Yangian invariant $\Psi_{8,4}$ of the algebra $\mathfrak{u}(2,2 \mid 0+4)$ to a $\mathrm{U}(2)$ integral by making use of the bosonic delta functions. We display the resulting integral here only for equal integer deformation parameters $c_{1}=c_{2}=c_{3}=c_{4}$ in order to spare the reader a slightly more cumbersome expression. In addition, we abbreviate frequently occurring differences of complex deformation parameters as $z_{1} \equiv v_{1}-v_{2}, z_{2} \equiv v_{2}-v_{3}, z_{3} \equiv v_{3}-v_{4}$. This yields

$$
\begin{aligned}
\Psi_{8,4}= & \frac{\delta^{4 \mid 0}(P) \delta^{0 \mid 8}(Q)}{s_{1234}^{8}} \int_{\mathrm{U}(2)}[\mathrm{d} \mathcal{U}] \frac{\delta^{0 \mid 8}(\mathrm{a} \mathcal{U}+\mathrm{b})}{\operatorname{det} \mathcal{U}^{2-c_{4}}\left|\mathrm{E}_{1} \operatorname{det}\left(\mathcal{U}-\mathrm{F}_{1}\right)\right|^{2\left(1+z_{1}\right)}} \\
& \cdot \frac{1}{\left|\mathrm{E}_{2} \operatorname{det}\left(\mathcal{U}-\mathrm{F}_{2}\right)\right|^{2\left(1+z_{2}\right)}\left|\mathrm{E}_{3} \operatorname{det}\left(\mathcal{U}-\mathrm{F}_{3}\right)\right|^{2\left(1+z_{3}\right)}} .
\end{aligned}
$$


Here the momentum and supermomentum conserving delta functions are defined in (2.8). In the denominator, we rearranged the minors of the Graßmannian integrand (3.13) into $2 \times 2$ determinants depending on the kinematic data

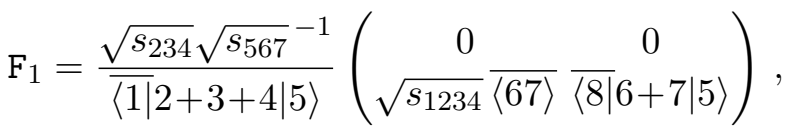

$$
\begin{aligned}
& \mathrm{E}_{1}=\frac{\overline{\langle 1}|2+3+4| 5\rangle}{s_{1234}} \\
& \mathrm{~F}_{2}=\frac{{\sqrt{s_{234} s_{567}}}^{-1}}{\overline{\langle 12\rangle}\langle 56\rangle}\left(\begin{array}{cc}
\langle 43\rangle \overline{\langle 1|} 2+3+4 \mid 5+6 \overline{|7\rangle} & \sqrt{s_{1234}}\langle 34\rangle\langle 56\rangle \overline{\langle 18\rangle} \\
\sqrt{s_{1234}} \overline{\langle 2|} 3+4 \mid 5+6 \overline{|7\rangle} & \langle 56\rangle \overline{\langle 2|3+4| 5+6+7 \mid 8\rangle}
\end{array}\right), \quad \mathrm{E}_{2}=\frac{\overline{\langle 12\rangle}\langle 56\rangle}{s_{1234}}, \\
& \mathrm{~F}_{3}=\frac{\sqrt{s_{567}} \sqrt{s_{234}}-1}{\overline{\langle 4|} 5+6+7|8\rangle}\left(\begin{array}{ll}
0 & \sqrt{s_{1234}}\langle 23\rangle \\
0 & \overline{\langle 4|} 2+3|1\rangle
\end{array}\right), \quad \mathrm{E}_{3}=\frac{\overline{\langle 4|} 1+2+3|8\rangle}{s_{1234}} .
\end{aligned}
$$

The numerator involves further kinematics contained in

$$
\begin{aligned}
& \mathrm{a}=\frac{1}{\sqrt{s_{234}}}\left(\begin{array}{c}
\left.\sqrt{s_{1234}}(\overline{\langle 34}\rangle \tilde{\eta}^{2}+\overline{\langle 42\rangle} \tilde{\eta}^{3}+\overline{\langle 23\rangle} \tilde{\eta}^{4}\right) \\
s_{234} \tilde{\eta}^{1}+\left\langle 2|3+4| \overline{1\rangle} \tilde{\eta}^{2}+\left\langle 3|2+4| \overline{1\rangle} \tilde{\eta}^{3}+\left\langle 4|2+3| \overline{1\rangle} \tilde{\eta}^{4}\right.\right.\right.
\end{array}\right)^{t}, \\
& \mathrm{~b}=\frac{1}{\sqrt{s_{567}}}\left(\begin{array}{c}
\sqrt{s_{5678}}\left(\overline{\langle 67\rangle} \tilde{\eta}^{5}+\overline{\langle 75\rangle} \tilde{\eta}^{6}+\overline{\langle 56\rangle} \tilde{\eta}^{7}\right) \\
\left\langle 5|6+7| \overline{|8\rangle} \tilde{\eta}^{5}+\langle 6|5+7| 8\rangle \tilde{\eta}^{6}+\left\langle 7|5+6| \overline{|8\rangle} \tilde{\eta}^{7}+s_{567} \tilde{\eta}^{8}\right.\right.
\end{array}\right)^{t}
\end{aligned}
$$

with the four-dimensional fermionic variables $\tilde{\eta}^{i}=\left(\tilde{\eta}_{\dot{a}}^{i}\right)$. The $\mathrm{U}(2)$ integral for the deformed Yangian invariant $\Psi_{8,4}$ in (4.15) is structurally a straightforward generalization of the U(1) integral for $\Psi_{6,3}$ with $c_{1}=c_{2}=c_{3}$ from (4.4). Most characteristically, the absolute values in the denominator contain $2 \times 2$ determinants in the $\mathrm{U}(2)$ case, and there is one additional such factor compared to the $\mathrm{U}(1)$ integral.

Despite the structural similarities, the two integrals behave fundamentally different in the undeformed limit, where $c_{i}=0$ and all $v_{i}$ are equal, i.e. $z_{i}=0$. The $\mathrm{U}(1)$ integral (4.4) computing $\Psi_{6,3}$ reduces to the contour integral (4.6), which is finite for generic kinematics and can be evaluated by means of the residue theorem. Divergencies may arise for special kinematic configurations, such as $s_{234}=0$ or $s_{345}=0$ where poles of the integrand move onto the integration contour, cf. (4.7). In the U(2) integral (4.15) for $\Psi_{8,4}$, we can safely set $c_{i}=0$. However, upon imposing $z_{i}=0$, we observe that the integral diverges even for generic kinematics.

Let us sketch this in more detail. To gain a handle on the divergencies, we first need a criterion for which kinematic data the integrand of $\Psi_{8,4}$ in (4.15) can become singular:

$$
\operatorname{det}\left(\mathcal{U}-\mathrm{F}_{i}\right)=0 \quad \text { for some } \mathcal{U} \in \mathrm{U}(2) \quad \Leftrightarrow \quad \operatorname{det}\left(\mathrm{F}_{i} \mathrm{~F}_{i}^{\dagger}-1_{2}\right) \leq 0,
$$

see e.g. theorem 5.5.1 in [62]. For the matrices from (4.16), this criterion reads ${ }^{7}$

$$
\operatorname{det}\left(\mathrm{F}_{1} \mathrm{~F}_{1}^{\dagger}-1_{2}\right) \propto-s_{2345}, \quad \operatorname{det}\left(\mathrm{F}_{2} \mathrm{~F}_{2}^{\dagger}-1_{2}\right) \propto s_{3456}, \quad \operatorname{det}\left(\mathrm{F}_{3} \mathrm{~F}_{3}^{\dagger}-1_{2}\right) \propto-s_{4567},
$$

\footnotetext{
${ }^{7}$ These variables are combinations of dual conformal cross ratios before dropping the factors. Recall in this context that also the analogous quantities for $\Psi_{6,3}$ in (4.7) are such cross ratios.
} 
where we dropped non-negative factors. The signs of these four-particle Mandelstam variables determine which factors of the integrand in (4.15) become singular while the integration variable $\mathcal{U}$ traverses the $\mathrm{U}(2)$ group manifold. They give rise to $2^{3}=8$ kinematic regions. Clearly, the integrand contains singularities in seven of these. Based on a numerical study using random momenta, the only non-singular region $s_{2345}<0, s_{3456}>0$, $s_{4567}<0$ appears to be kinematically forbidden for our choice of energy signs in (3.1). In the undeformed limit $c_{i}=0$ and $z_{i} \rightarrow 0$, the singularities of the integrand yield a divergent integral (4.15) for $\Psi_{8,4}$. Presently, we are able to extract the leading divergent terms ${ }^{8}$ from this integral by focusing on the vicinity of the singularities [65]. We find that the precise expressions of these terms depend on a rich substructure of the kinematic regions (4.19). These subregions are defined primarily using the signs of $s_{345}, s_{456}, s_{781}, s_{812}$, and those of certain determinants of Mandelstam variables. The consecutive three-particle Mandelstam variables appearing here and the four-particle ones in (4.19) are precisely all those whose signs are allowed to change in our setup. In most subregions the leading divergent terms are of the form $\frac{1}{z_{i}}$, while in some there exist even more singular terms with $\frac{1}{z_{i} z_{j}}$.

The occurrence of these divergent terms signals that we cannot simply take the undeformed limit of the Yangian invariant $\Psi_{8,4}$ in (4.15) and end up with a finite expression that agrees with the amplitude $\mathcal{A}_{8,4}$ in one kinematic region. Finding a way to extract this amplitude from $\Psi_{8,4}$ is arguably one of the most pressing open problems of our unitary Graßmannian integral approach. On the one hand, it certainly requires more sophisticated techniques for the evaluation of the $\mathrm{U}(2)$ integral (4.15). It could be helpful use a matrix version of the Cayley transformation to map the $\mathrm{U}(2)$ integration variable $\mathcal{U}$ to a Hermitian $2 \times 2$ matrix, see e.g. [66]. This matrix can in turn be mapped as in (2.1) to a vector in real Minkowski space $\mathbb{R}^{1,3}$. Our integral (4.15) then essentially takes the form of a oneloop Feynman integral with complex exponents of the propagators and complex external momenta given in terms of the matrices $\mathrm{F}_{i}$. This might make the machinery developed for such Feynman integrals applicable to our problem. On the other hand, also conceptually the relation between $\Psi_{8,4}$ and $\mathcal{A}_{8,4}$ needs further attention. In this regard, we refer the reader to section 6.2 below. There we will demonstrate the existence of a divergent term, albeit only for special kinematics, in case of the simple invariant $\Psi_{4,2}$ from section 4.1. This will suggest a new perspective even for the relation between $\Psi_{4,2}$ and $\mathcal{A}_{4,2}$.

Although the exact connection between the Yangian invariants $\Psi_{2 K, K}$ computed by the unitary Graßmannian integral (3.5) and the amplitudes $\mathcal{A}_{2 K, K}$ is still unclear for general $K$, we can establish a fairly complete understanding of $\Psi_{2 K, K}$ from the point of view of integrability. We will develop it systematically in the following sections. For this we will employ a different basis, in which the Yangian invariants are expressed in terms of harmonic oscillators instead of spinor helicity variables. These new variables are instrumental in revealing fascinating relations between the unitary Graßmannian integral and other subjects such as integrable spin chains and matrix models. The inquisitive reader may already jump ahead and take a first look at table 1, where different interpretations of our unitary integral are summarized.

\footnotetext{
${ }^{8}$ We are grateful to Jacob Bourjaily for supplying us with data adapted from $[63,64]$ to cross-check this result.
} 


\section{Graßmannian integral in oscillator basis}

In this section, we define an analogue of the unitary Graßmannian integral (3.5) for a class of representations that are constructed using oscillator algebras instead of spinor helicity variables. This integral formula reveals interesting connections between Yangian invariants and matrix models. Moreover, we show that it is related to the original formula (3.5) via a Bargmann transformation. This integral transformation generalizes the change of basis from Fock to position space for the harmonic oscillator in elementary quantum mechanics.

\section{$5.1 \quad$ Oscillator representations}

We introduce oscillator representations of the non-compact superalgebra $\mathfrak{u}(p, q \mid r+s)$ following [52]. Such representations have a long history in the physics literature and are sometimes referred to as "ladder representations", see e.g. [51]. In case of the algebra $\mathfrak{u}(2,2 \mid 4)$, they are discussed also in [67] and play a prominent role in the planar $\mathcal{N}=4$ SYM spectral problem. For $\mathfrak{u}(p, q=p \mid r+s)$, they are unitarily equivalent to the representations that we encountered already in section 3.4, as will be shown in section 5.4 below. This justifies our use of the symbols $\mathscr{D}_{c}$ and $\overline{\mathscr{D}}_{c}$ for the two classes of representations defined in the following.

The basic ingredient of both classes is a family of superoscillators obeying

$$
\left[\mathbf{A}_{\mathcal{A}}, \overline{\mathbf{A}}_{\mathcal{B}}\right\}=\delta_{\mathcal{A B}}, \quad\left[\mathbf{A}_{\mathcal{A}}, \mathbf{A}_{\mathcal{B}}\right\}=0, \quad\left[\overline{\mathbf{A}}_{\mathcal{A}}, \overline{\mathbf{A}}_{\mathcal{B}}\right\}=0, \quad \mathbf{A}_{\mathcal{A}}^{\dagger}=\overline{\mathbf{A}}_{\mathcal{A}}, \quad \mathbf{A}_{\mathcal{A}}|0\rangle=0
$$

where the indices of the annihilation operators $\mathbf{A}_{\mathcal{A}}$ and creation operators $\overline{\mathbf{A}}_{\mathcal{A}}$ take the values $\mathcal{A}=1, \ldots, n+m$ with $n=p+q$ and $m=r+s$. It is equipped with a conjugation $\dagger$ and acts on a Fock space $\mathcal{F}$ that is spanned by monomials in $\overline{\mathbf{A}}_{\mathcal{A}}$ acting on a vacuum state $|0\rangle$. We illustrate the grading of the superindex $\mathcal{A}$ by means of the creation operators,

$$
\left(\overline{\mathbf{A}}_{\mathcal{A}}\right)=\left(\begin{array}{c}
\overline{\mathbf{A}}_{\mathrm{A}} \\
\cdots \cdots \\
\cdots \overline{\mathbf{A}}_{\dot{\mathrm{A}}}
\end{array}\right)=\left(\begin{array}{c}
\overline{\mathbf{a}}_{\alpha} \\
\overline{\mathbf{c}}_{a} \\
\overline{\mathbf{b}}_{\dot{\alpha}} \\
\cdots \ldots \\
\overline{\mathbf{d}}_{\dot{a}}
\end{array}\right) .
$$

Here we first split the family of superoscillators with $\mathfrak{g l}(n \mid m)$ index $\mathcal{A}$ into two parts. One carries a $\mathfrak{g l}(p \mid r)$ index $\mathrm{A}=1, \ldots, p+r$ and the other one a $\mathfrak{g l}(q \mid s)$ index $\dot{\mathrm{A}}=p+r+1, \ldots, n+$ $m$. Then we spelled out the superoscillators $\overline{\mathbf{A}}_{\mathrm{A}}, \overline{\mathbf{A}}_{\dot{\dot{A}}}$ in terms of bosonic oscillators $\overline{\mathbf{a}}_{\alpha}, \overline{\mathbf{b}}_{\dot{\alpha}}$ and fermionic oscillators $\overline{\mathbf{c}}_{a}, \overline{\mathbf{d}}_{\dot{a}}$ with the index ranges $\alpha=1, \ldots, p, \dot{\alpha}=1, \ldots, q, a=$ $1, \ldots, r$, and $\dot{a}=1, \ldots, s$. This notation fixes the grading and is inspired by [35] and [46].

A set of generators $\mathbf{J}_{\mathcal{A B}}$ of the $\mathfrak{g l}(n \mid m)$ superalgebra is provided by

$$
\left(\mathbf{J}_{\mathcal{A B}}\right)=\left(\begin{array}{c:c}
\overline{\mathbf{A}}_{\mathrm{A}} \mathbf{A}_{\mathrm{B}} & \overline{\mathbf{A}}_{\mathrm{A}} \overline{\mathbf{A}}_{\dot{\mathrm{B}}} \\
\hdashline-(-1)^{|\dot{\mathrm{A}}|} \mathbf{A}_{\dot{\mathrm{A}}} \mathbf{A}_{\mathrm{B}} & -(-1)^{||^{|\dot{\mathrm{A}}|}} \mathbf{A}_{\dot{\mathrm{A}}} \overline{\mathbf{A}}_{\dot{\mathrm{B}}}
\end{array}\right) .
$$


The two diagonal blocks of this supermatrix realize the subalgebras $\mathfrak{g l}(p \mid r)$ and $\mathfrak{g l}(q \mid s)$, respectively. Let $\mathscr{D}_{c} \subset \mathcal{F}$ be the eigenspace of the central element

$$
\mathbf{C}=\operatorname{tr}\left(\mathbf{J}_{\mathcal{A B}}\right)
$$

with eigenvalue $c$. This infinite-dimensional space forms a unitary representation of the superalgebra $\mathfrak{u}(p, q \mid r+s)$ for each $c \in \mathbb{Z}$, see [52]. It contains a lowest weight state annihilated, by definition, by all generators $\mathbf{J}_{\mathcal{A B}}$ with $\mathcal{A}>\mathcal{B}$. Note that the space $\mathscr{D}_{c}$ is finite-dimensional in the special cases $q=0$ or $p=0$. We define a class of dual representations by applying the automorphism $\overline{\mathbf{J}}_{\mathcal{A B}}=-(-1)^{|\mathcal{A}|+|\mathcal{A}||\mathcal{B}|} \mathbf{J}_{\mathcal{A B}}^{\dagger}$ of the $\mathfrak{g l}(n \mid m)$ algebra to the generators in (5.3),

$$
\left(\overline{\mathbf{J}}_{\mathcal{A B}}\right)=\left(\begin{array}{c:c}
-(-1)^{|\mathrm{A}|+|\mathrm{A}||\mathrm{B}|} \overline{\mathbf{A}}_{\mathrm{B}} \mathbf{A}_{\mathrm{A}} & -(-1)^{|\mathrm{A}|+|\dot{\mathrm{B}}|+|\mathrm{A}||\dot{\mathrm{B}}|} \mathbf{A}_{\dot{\mathrm{B}}} \mathbf{A}_{\mathrm{A}} \\
\hdashline(-1)^{|\dot{\mathrm{A}}|+|\dot{\mathrm{A}}||\mathrm{B}|} \overline{\mathbf{A}}_{\mathrm{B}} \overline{\mathbf{A}}_{\dot{\mathrm{A}}} & (-1)^{|\dot{\mathrm{A}}|+|\dot{\mathrm{B}}|+|\dot{\mathrm{A}}||\dot{\mathrm{B}}|} \mathbf{A}_{\dot{\mathrm{B}}} \overline{\mathbf{A}}_{\dot{\mathrm{A}}}
\end{array}\right) .
$$

We denote by $\overline{\mathscr{D}}_{c} \subset \mathcal{F}$ the eigenspace of the central element

$$
\overline{\mathbf{C}}=\operatorname{tr}\left(\overline{\mathbf{J}}_{\mathcal{A B}}\right)
$$

with eigenvalue $c$. This space carries a unitary representation of $\mathfrak{u}(p, q \mid r+s)$ for each $c \in \mathbb{Z}$. It contains a highest weight state annihilated by all $\overline{\mathbf{J}}_{\mathcal{A B}}$ with $\mathcal{A}<\mathcal{B}$.

Let us add that, recently, all unitary representations of the superalgebra $\mathfrak{s u}(p, q \mid m)$ were constructed by means of a generalized oscillator formalism in [68]. This paper also contains an informative overview of the evolution of the oscillator method with numerous further references.

\subsection{Graßmannian integral}

The oscillator representations of $\mathfrak{u}(p, q \mid r+s)$ from the previous section at hand, we construct an analogue of the unitary Graßmannian integral (3.5). Yangian invariants for these representations are given by

$$
\left|\Psi_{2 K, K}\right\rangle=\int_{\mathrm{U}(K)}[\mathrm{d} \mathcal{C}] \mathscr{F}(\mathcal{C})(\operatorname{det} \mathcal{C})^{r} e^{\operatorname{tr}\left(\mathbf{I}_{\bullet} \mathcal{C}^{\dagger}+\mathcal{C} \mathbf{I}_{\circ}^{t}\right)}|0\rangle .
$$

Here the delta functions of (3.5) have been replaced by an exponential function acting on a Fock vacuum. Its argument contains the $K \times K$ matrices

$$
\mathbf{I}_{\circ}=\left(\begin{array}{ccc}
(1: K+1) & \cdots & (1: 2 K) \\
\vdots & & \vdots \\
(K: K+1) & \cdots & (K: 2 K)
\end{array}\right)
$$

Their entries are contractions of creation operators,

$$
(k \bullet l)=\sum_{\mathrm{A}} \overline{\mathbf{A}}_{\mathrm{A}}^{l} \overline{\mathbf{A}}_{\mathrm{A}}^{k}, \quad(k \circ l)=\sum_{\dot{\mathrm{A}}} \overline{\mathbf{A}}_{\dot{\mathrm{A}}}^{l} \overline{\mathbf{A}}_{\dot{\mathrm{A}}}^{k} .
$$


They are $\mathfrak{g l}(p \mid r)$ and $\mathfrak{g l}(q \mid s)$ invariant, respectively. Moreover, they are bosonic because fermionic oscillators appear only in quadratic terms. The rest of (3.5) can be found essentially unchanged in $(5.7)$. The function $\mathscr{F}(\mathcal{C})$ is the manifestly single-valued expression from (3.13), which we obtained from the formal integrand in (3.6), now in general with $q \neq p$. It contains the inhomogeneities $v_{i}$ and the representation labels $c_{i}$. These are related via (3.7) to the deformation parameters $v_{i}^{ \pm}$, which have to obey (3.8). The $v_{i}$ and $c_{i}$ also enter the monodromy matrix $M(u)$ defined in (2.11) that is associated with $\left|\Psi_{2 K, K}\right\rangle$ from (5.7). The first $K$ of its $N=2 K$ sites carry the oscillator representations $\overline{\mathscr{D}}_{c_{i}}$, and the remaining $K$ sites have the representations $\mathscr{D}_{c_{i}}$. Therefore the $\mathfrak{g l}(n \mid m)$ generators in the Lax operators $(2.9)$ of the monodromy are

$$
J_{\mathcal{A B}}^{i}= \begin{cases}\overline{\mathbf{J}}_{\mathcal{A B}}^{i} & \text { for } \quad i=1, \ldots, K, \\ \mathbf{J}_{\mathcal{A B}}^{i} & \text { for } \quad i=K+1, \ldots, 2 K,\end{cases}
$$

which are given in (5.5) and (5.3), respectively. With this monodromy $M(u)$, the state $\left|\Psi_{2 K, K}\right\rangle$ satisfies the Yangian invariance condition (2.15) and therefore also (2.16). A proof of this central statement is presented in [1]. It is a straightforward extension of the proof for the bosonic algebra $\mathfrak{u}(p, q)$ in [69]. In the latter reference, the existence of a closed contour is assumed to perform partial integrations, and a measure of the form (3.9) is used. However, at the time, no explicit example of such a contour was known. This gap is filled here by the unitary contour and the single-valued integrand, recall section 3.3.

We will show in section 5.4 that the Yangian invariants $\left|\Psi_{2 K, K}\right\rangle$ in (5.7) are related to the $\Psi_{2 K, K}$ in (3.5) by a change of basis for representations of the algebra $\mathfrak{u}(p, q=p \mid r+s)$. In case of $\mathfrak{u}(2,2 \mid 4)$ and vanishing deformation parameters $v_{i}^{ \pm} \rightarrow 0$, these invariants are of relevance for the $\mathcal{N}=4 \mathrm{SYM}$ amplitudes $\mathcal{A}_{2 K, K}$, as we argued in section 4 . Is there also a significance of the $\left|\Psi_{2 K, K}\right\rangle$ directly in the oscillator basis? As is turns out, their integral representation (5.7) reduces to known matrix models for specific values of $v_{i}^{ \pm}$. Equation (3.8) constraining the $v_{i}^{ \pm}$has a solution where all minors appearing in $\mathscr{F}(\mathcal{C})$ from (3.13), except for $\operatorname{det} \mathcal{C}$, have a vanishing exponent,

$$
\mathscr{F}(\mathcal{C})=(\operatorname{det} \mathcal{C})^{-r+q-s-c_{K}}
$$

With this integrand, (5.7) is equivalent to the Leutwyler-Smilga model [70], which describes aspects of quantum chromodynamics in a certain low energy regime. For $q=s+c_{K}$, all factors of $\operatorname{det} \mathcal{C}$ disappear from (5.7), and it becomes the Brezin-Gross-Witten model [71-73], which appears in the context of two-dimensional lattice gauge theory. Remarkably, these integrals can be computed exactly as determinants of matrices whose entries are Bessel functions. For two independent matrices $\mathbf{I}_{\bullet}^{t}$ and $\mathbf{I}_{\circ}$, this was achieved in [74] using the character expansion methods of [75]. This determinant formula will allow us in section 7 below to establish a heuristic yet intriguing link between $\left|\Psi_{4,2}\right\rangle$ and the cusp equation [76, 77], which governs certain all-loop results in planar $\mathcal{N}=4 \mathrm{SYM}$. In addition, the two aforementioned matrix integrals provide solutions, so-called $\tau$-functions, of the Kadomtsev-Petviashvili (KP) hierarchy, cf. [78, 79]. It would be interesting to expose a connection between the well-studied integrable structure of this hierarchy, see the substantial review in [80], and 


\begin{tabular}{|l|l|}
\hline \multicolumn{1}{|c|}{ Parameters } & \multicolumn{1}{c|}{ Interpretation } \\
\hline$v_{i}^{ \pm} \rightarrow 0$ and $\mathfrak{u}(2,2 \mid 4)$ & $\begin{array}{l}\Psi_{2 K, K} \text { related to amplitude } \mathcal{A}_{2 K, K} ? \\
(\text { section } 4)\end{array}$ \\
\hline$v_{i}^{ \pm}$s.t. $\mathscr{F}(\mathcal{C})(\operatorname{det} \mathcal{C})^{r}=\left\{\begin{array}{l}(\operatorname{det} \mathcal{C})^{q-s-c_{K}} \\
1\end{array}\right.$ & $\begin{array}{l}\left|\Psi_{2 K, K}\right\rangle \text { is }\left\{\begin{array}{l}\text { Leutwyler-Smilga model [70] } \\
\text { Brezin-Gross-Witten model [71-73] }\end{array}\right. \\
\text { Link to cusp equation for }\left|\Psi_{4,2}\right\rangle \text { (section 7) }\end{array}$ \\
\hline$v_{i}^{ \pm}$general & $\begin{array}{l}\left|\Psi_{2 K, K}\right\rangle \text { equals product of R-matrices } \\
(\text { section } 6 \text { and appendix B) }\end{array}$ \\
\hline
\end{tabular}

Table 1. Interpretations of the unitary Graßmannian integrals (3.5) and (5.7) for different deformation parameters $v_{i}^{ \pm}$in the integrand $\mathscr{F}(\mathcal{C})$.

the Yangian invariance of the integrals. Moreover, we are tempted to speculate that (5.7) could be a KP $\tau$-function for a wider range of the deformation parameters $v_{i}^{ \pm}$. Let us move on to another interpretation of the $\left|\Psi_{2 K, K}\right\rangle$ that clarifies their role within the QISM and is valid even for general $v_{i}^{ \pm}$obeying (3.8). We will discuss in section 6 below that the Yangian invariant $\left|\Psi_{4,2}\right\rangle$ can be understood as an R-matrix. Furthermore, in appendix B we show that $\left|\Psi_{2 K, K}\right\rangle$ from (5.7) with its $\mathrm{U}(K)$ contour can be constructed by "gluing" together multiple copies of $\left|\Psi_{4,2}\right\rangle$ with $\mathrm{U}(2)$ contours. Thus $\left|\Psi_{2 K, K}\right\rangle$ corresponds to a product of R-matrices. The interpretations outlined in this paragraph are summarized in table 1.

\subsection{Bargmann realization and transformation}

The equivalence of the two Graßmannian integral formulas (3.5) and (5.7) will be established in the next section using a Bargmann transformation. For the one-dimensional harmonic oscillator in quantum mechanics, this integral transformation implements the change of basis between Fock and position space. We introduce the Bargmann transformation along the lines of the original publication [81]. From the outset, we work in a multi-dimensional setting because it is needed for our application.

We start out with a family of bosonic oscillators on a Fock space obeying

$$
\left[\mathbf{a}_{\alpha}, \overline{\mathbf{a}}_{\beta}\right]=\delta_{\alpha \beta}, \quad \mathbf{a}_{\alpha}^{\dagger}=\overline{\mathbf{a}}_{\alpha}, \quad \mathbf{a}_{\alpha}|0\rangle=0
$$

with $\alpha, \beta=1, \ldots, p$. Let $\mathbf{a}=\left(\mathbf{a}_{\alpha}\right)$ etc. denote $r$-component column vectors. The relations in (5.12) are implemented by the Bargmann realization

$$
\overline{\mathbf{a}} \mapsto z, \quad \mathbf{a} \mapsto \partial_{z}, \quad|0\rangle \mapsto \Psi_{0}(z)=1
$$

on the Bargmann space $\mathscr{H}_{\mathrm{B}}$. This is the Hilbert space of holomorphic functions of $z \in \mathbb{C}^{p}$ with the inner product

$$
\langle\Psi(z), \Phi(z)\rangle_{\mathrm{B}}=\int_{\mathbb{C}^{p}} \frac{\mathrm{d}^{p} \bar{z} \mathrm{~d}^{p} z}{(2 \pi i)^{p}} e^{-\bar{z}^{t} z} \overline{\Psi(z)} \Phi(z),
$$


where $(2 i)^{-p} \mathrm{~d}^{p} \bar{z} \mathrm{~d}^{p} z=\mathrm{d}^{p} \operatorname{Re} z \mathrm{~d}^{p} \operatorname{Im} z$ is understood as the measure on $\mathbb{R}^{2 p}$. In particular, this inner product implements the reality condition in (5.12), i.e. $\partial_{z_{\alpha}}^{\dagger}=z_{\alpha}$. The Bargmann realization can be thought of as a concrete implementation of the formal Fock space operators. For recent expositions of this realization see e.g. [82, 83], where it is called "holomorphic representation".

In addition, we introduce another family of canonical variables obeying different reality conditions,

$$
\left[\partial_{x_{\alpha}}, x_{\beta}\right]=\delta_{\alpha \beta}, \quad \partial_{x_{\alpha}}^{\dagger}=-\partial_{x_{\alpha}}, \quad x_{\alpha}^{\dagger}=x_{\alpha} .
$$

These are considered as operators on the Hilbert space $\mathscr{H}_{\text {Sch }}$ of square integrable functions of the variable $x \in \mathbb{R}^{p}$ with the inner product

$$
\langle\Psi(x), \Phi(x)\rangle_{\mathrm{Sch}}=\int_{\mathbb{R}^{p}} \mathrm{~d}^{p} x \overline{\Psi(x)} \Phi(x) .
$$

This implementation of (5.15) is referred to as Schrödinger realization. For the example of the one-dimensional harmonic oscillator, it may be interpreted as the realization in position space.

We observe that by a naive counting the degrees of freedom in $\mathscr{H}_{\mathrm{B}}$ and $\mathscr{H}_{\text {Sch }}$ do match. A function $\Psi(z)$ in $\mathscr{H}_{\mathrm{B}}$ depends on $p$ complex coordinates $z_{\alpha}$ but not on their complex conjugates $\bar{z}_{\alpha}$. Similarly, $\Psi(x)$ in $\mathscr{H}_{\text {Sch }}$ is a function of $p$ real coordinates $x_{\alpha}$. Thus we want to identify the canonical variables in $\mathscr{H}_{\mathrm{B}}$ and $\mathscr{H}_{\text {Sch. }}$. For this purpose we make the ansatz

$$
\partial_{z} \leftrightarrow A\left(x+\gamma \partial_{x}\right), \quad z \leftrightarrow \bar{A}\left(x-\gamma \partial_{x}\right)
$$

where we allow for a constant $\gamma>0$ and a $p \times p$ matrix $A$, whose complex conjugate we denote $\bar{A}$. The second relation is obtained from the first one by taking the Hilbert space adjoint. For (5.17) to be compatible with the commutation relations and reality conditions in (5.12) and (5.15), we have to impose

$$
2 \gamma A A^{\dagger}=1_{p},
$$

where ${ }^{\dagger}$ stands for Hermitian conjugation of matrices. Note that this condition can be solved trivially by taking $A \propto 1_{p}$, in which case the components of the relations in (5.17) decouple. The identification (5.17) of the Hilbert spaces $\mathscr{H}_{\mathrm{B}}$ and $\mathscr{H}_{\text {Sch }}$ is implemented by the Bargmann transformation

$$
\Psi(z)=\langle\overline{\mathcal{K}(z, x)}, \Psi(x)\rangle_{\mathrm{Sch}}, \quad \Psi(x)=\langle\mathcal{K}(z, x), \Psi(z)\rangle_{\mathrm{B}}
$$

with the kernel

$$
\mathcal{K}(z, x)=(\pi \gamma)^{-\frac{p}{4}} e^{-\gamma z^{t} A A^{t} z-\frac{1}{2 \gamma} x^{t} x+2 z^{t} A x} .
$$

This kernel solves the differential equations obtained by imposing (5.17) on (5.19),

$$
\partial_{z} \mathcal{K}(z, x)=A\left(x-\gamma \partial_{x}\right) \mathcal{K}(z, x), \quad z \mathcal{K}(z, x)=\bar{A}\left(x+\gamma \partial_{x}\right) \mathcal{K}(z, x) .
$$


The prefactor in (5.20) is fixed by demanding that the transformation (5.19) preserves the unit norm of the vacuum state $\Psi_{0}(x)=(\pi \gamma)^{-\frac{p}{4}} e^{-\frac{1}{2 \gamma} x^{t} x}$. Equation (5.19) together with the definitions of the inner products in (5.14) and (5.16) yields the concrete form of the Bargmann transformation as an integral transformation that we will use in the following section.

We also briefly discuss a realization of the fermionic oscillator algebra

$$
\left\{\mathbf{c}_{a}, \overline{\mathbf{c}}_{b}\right\}=\delta_{a b}, \quad \mathbf{c}_{a}^{\dagger}=\overline{\mathbf{c}}_{a}, \quad \mathbf{c}_{a}|0\rangle=0,
$$

where $a, b=1, \ldots, r$. It is realized on a Graßmann algebra with $r$ variables $\eta_{a}$,

$$
\overline{\mathbf{c}} \mapsto \eta, \quad \mathbf{c} \mapsto \partial_{\eta}, \quad|0\rangle \mapsto 1 .
$$

On order to implement the adjoint in (5.22) as $\eta_{a}^{\dagger}=\partial_{\eta_{a}}$, one has to define an inner product on functions $\Psi(\eta)$. This can be done in formal analogy to (5.14) of the Bargmann realization. The integral is replaced by a Berezin integral, and instead of complex conjugation one uses an antiinvolution of the Graßmann algebra. Consequently, (5.23) can be understood as a fermionic Bargmann realization, see e.g. [82, 83] for more details.

\subsection{Transformation of generators and integral}

Utilizing the tools from the previous section, in particular the Bargmann transformation, we now identify the $\mathfrak{u}(p, q=p \mid r+s)$ representations in terms of spinor helicity variables from section 3.4 with those defined using oscillators in section 5.1. This then allows us to establish the equivalence of the two unitary Graßmannian integral formulas (3.5) and (5.7) for these algebras. The identification of the representations goes back to a calculation for the special case of the conformal algebra $\mathfrak{s u}(2,2)[49]$. In this case, it can be associated with a transformation from a Lorentz to a maximally compact basis of the algebra. The reason being that the diagonal blocks of the matrices of spinor helicity (3.14) and oscillator (5.3) generators are associated with the corresponding subalgebras $\mathfrak{s l}\left(\mathbb{C}^{2}\right)$ and $\mathfrak{s u}(2) \times \mathfrak{s u}(2) \times \mathfrak{u}(1)$, respectively. In addition, the identification of representations is of particular importance in the $\mathfrak{u}(2,2 \mid 4)$ case because it provides a connection between the variables frequently used for the $\mathcal{N}=4 \mathrm{SYM}$ amplitudes $\mathcal{A}_{N, K}$ and those featuring in the spectral problem of this theory.

To begin with, we concentrate on the positive energy representations $\mathscr{D}_{c}$ of $\mathfrak{u}(p, p \mid r+s)$. Our aim is to relate the generators $\mathbf{J}_{\mathcal{A B}}$ from (5.3) to the $\mathfrak{J}_{\mathcal{A B}}$ from (3.14). In a first step, we employ the bosonic (5.13) and the fermionic (5.23) Bargmann realizations for the oscillators appearing in the former set of generators. In a second step, we apply the replacement (5.17) to the bosonic variables, while the fermionic ones remain unchanged. For the creation operators (5.2), this reads

$$
\left(\overline{\mathbf{A}}_{\mathcal{A}}\right)=\left(\begin{array}{c}
\overline{\mathbf{a}}_{\alpha} \\
\cdots \ldots \\
\overline{\mathbf{c}}_{a} \\
\overline{\mathbf{b}}_{\dot{\alpha}} \\
\overline{\mathbf{d}}_{\dot{a}}
\end{array}\right) \mapsto\left(\begin{array}{c}
z_{\alpha} \\
\cdots \ldots \\
\eta_{a} \\
\hdashline w_{\dot{\alpha}} \\
\hdashline \tilde{\eta}_{\dot{a}}
\end{array}\right) \leftrightarrow\left(\begin{array}{c}
\frac{1}{\sqrt{2}}\left(\lambda_{\alpha}-\partial_{\bar{\lambda}_{\alpha}}\right) \\
\eta_{a} \\
\frac{1}{\sqrt{2}}\left(\bar{\lambda}_{\alpha}-\partial_{\lambda_{\alpha}}\right) \\
\tilde{\eta}_{\dot{a}}
\end{array}\right)
$$


Recall the differing reality conditions of the superoscillators in (5.1) compared to those of the spinor helicity variables and fermions in (3.16). They allow to derive the transformation of the annihilation operators from (5.24). The replacement (5.17) is implemented by the Bargmann transformation (5.19). The latter is specified by

$$
\left(\begin{array}{c}
z_{\alpha} \\
\hdashline w_{\dot{\alpha}}
\end{array}\right) \in \mathbb{C}^{2 p}, \quad\left(\begin{array}{c}
x_{\alpha} \\
\hdashline y_{\dot{\alpha}}
\end{array}\right) \in \mathbb{R}^{2 p}, \quad A=\frac{1}{\sqrt{2}}\left(\begin{array}{c:c}
1_{p} & -i 1_{p} \\
\hdashline 1_{p} & i 1_{p}
\end{array}\right), \quad \gamma=\frac{1}{2},
$$

which are the arguments of holomorphic functions $\Psi(z, w)$ in the Bargmann space $\mathscr{H}_{\mathrm{B}}$, those of square integrable functions $\Psi(x, y)$ in $\mathscr{H}_{\text {Sch }}$, and the parameters of the transformation from (5.18), respectively. Importantly, to obtain spinor helicity variables, the $x, y \in \mathbb{R}^{p}$ are packaged into $\lambda=x+i y \in \mathbb{C}^{p}$. With these definitions, the Bargmann transformation (5.19) from $\mathscr{H}_{\mathrm{B}}$ to $\mathscr{H}_{\text {Sch }}$ takes the form

$$
\Psi(\lambda, \bar{\lambda})=\sqrt{\frac{2}{\pi}} e^{-\bar{\lambda}^{t} \lambda} \int_{\mathbb{C}^{2 p}} \frac{\mathrm{d}^{p} \bar{z} \mathrm{~d}^{p} z \mathrm{~d}^{p} \bar{w} \mathrm{~d}^{p} w}{(2 \pi i)^{2 p}} e^{-\bar{z}^{t} z-\bar{w}^{t} w-\bar{w}^{t} \bar{z}+\sqrt{2}\left(\bar{z}^{t} \lambda+\bar{w}^{t} \bar{\lambda}\right)} \Psi(z, w) .
$$

Notice that in this construction, the spinor helicity variables are a generalization of the position operator in the Schrödinger realization of the harmonic oscillator, cf. section 5.3. Next, we move on to the negative energy representations $\overline{\mathscr{D}}_{c}$ of $\mathfrak{u}(p, p \mid r+s)$, where we have to relate the generators $\overline{\mathbf{J}}_{\mathcal{A B}}$ from (5.5) to the $\overline{\mathfrak{J}}_{\mathcal{A B}}$ from (3.17). We proceed mostly analogous to above. The required changes can be summarized by replacements in the final transformation formulas (5.24), (5.26), and of the fermionic vacuum state,

$$
\lambda \mapsto \bar{\lambda}, \quad \eta \mapsto-\partial_{\eta}, \quad \partial_{\eta} \mapsto-\eta, \quad \tilde{\eta} \mapsto \partial_{\tilde{\eta}}, \quad \partial_{\tilde{\eta}} \mapsto \tilde{\eta}, \quad 1 \mapsto \eta_{1} \cdots \eta_{r} \tilde{\eta}_{1} \cdots \tilde{\eta}_{s} .
$$

The replacements of the fermions here can be realized by fermionic Fourier transformations.

Applying the transformation (5.24) in case of the representations $\mathscr{D}_{c}$ and together with (5.27) for $\overline{\mathscr{D}}_{c}$ yields the desired relations of the generators,

$$
\left(\mathbf{J}_{\mathcal{A B}}\right) \leftrightarrow D\left(\mathfrak{J}_{\mathcal{A B}}\right) D^{-1}, \quad\left(\overline{\mathbf{J}}_{\mathcal{A B}}\right) \leftrightarrow D\left(\overline{\mathfrak{J}}_{\mathcal{A B}}\right) D^{-1}
$$

with the supermatrix

$$
D=\left(\begin{array}{c:c:c:c}
1_{p} & 0 & 1_{p} & 0 \\
\hdashline 0 & \sqrt{2} 1_{r} & 0 & 0 \\
\hdashline-1_{p} & 0 & 1_{p} & 0 \\
\hdashline 0 & 0 & 0 & \sqrt{2} 1_{s}
\end{array}\right)
$$

whose grading can be inferred from (5.2). Consequently, the relations between the central elements (5.4) and (3.15) for the representations $\mathscr{D}_{c}$ together with those between (5.6) and (3.18) for $\overline{\mathscr{D}}_{c}$ read, respectively,

$$
\mathbf{C} \leftrightarrow \mathfrak{C}, \quad \overline{\mathbf{C}} \leftrightarrow \overline{\mathfrak{C}} .
$$


We remark that if the $\mathfrak{g l}(n \mid m)$ generators from (5.28) appear in Lax operators (2.9), the similarity transformation can be absorbed by a redefinition of the generators $E_{\mathcal{A B}}$. Thus we did not have to include such a similarity transformation in the specification of the monodromy matrices in (5.10) or (3.19).

Finally, we show the equivalence of the unitary Graßmannian integral formula (5.7) for $\left|\Psi_{2 K, K}\right\rangle$ and that in (3.5) for $\Psi_{2 K, K}$ in case of the algebras under consideration. This is achieved by applying the Bargmann realization to the superoscillators $\overline{\mathbf{A}}_{\mathcal{A}}^{i}$ and the vacuum $|0\rangle$ in $\left|\Psi_{2 K, K}\right\rangle$, as specified by the first replacement in (5.24). Next, we perform the Bargmann transformation (5.26) at all sites $i=1, \ldots, 2 K$. In both of these steps, we apply the replacement $(5.27)$ at the sites $i=1, \ldots, K$ with negative energy representations. The $\mathrm{U}(K)$ integral and the function $\mathscr{F}(\mathcal{C})$ in the Graßmannian formula (5.7) are unaffected by these operations. The exponential function in the integrand is transformed as

$$
(\operatorname{det} \mathcal{C})^{r} e^{\operatorname{tr}\left(\mathbf{I}_{\bullet} \mathcal{C}^{\dagger}+\mathcal{C} \mathbf{I}_{\circ}^{t}\right)}|0\rangle \leftrightarrow \delta_{\mathbb{C}}^{p K \mid 0}\left(C^{\perp} \boldsymbol{\lambda}\right) \delta^{0 \mid r K}\left(C^{\perp} \boldsymbol{\eta}\right) \delta^{0 \mid s K}(C \tilde{\boldsymbol{\eta}}),
$$

where we dropped an overall sign factor from rearranging the fermionic variables. This shows the equivalence of the two unitary Graßmannian integral formulas (5.7) and (3.5) for $\mathfrak{u}(p, p \mid r+s)$ Yangian invariants. Details on this calculation are provided in [1]. Let us sketch some key aspects. We split each of the matrices $\mathbf{I}_{\mathbf{8}}$, recall the definition of their entries in (5.9), into a sum of a matrix containing the bosonic oscillators and one with the fermionic ones. This leads to a factorization of the exponential function in (5.31). The Bargmann transformation of the factor with the bosonic variables becomes a highdimensional Gaußian integral. Its zero modes yield Fourier representations of the complex delta functions in (5.31). The series expansion of the exponential involving the fermionic variables truncates, which allows us to rewrite this exponential as fermionic delta functions.

\section{$6 \quad$ R-matrix and divergencies}

Until now, we have not directly evaluated the unitary Graßmannian integral (5.7) in the oscillator basis. We perform such a computation here for the sample invariant $\left|\Psi_{4,2}\right\rangle$ and establish its relation to the R-matrix. In particular, we demonstrate that the R-matrix of the Heisenberg spin chain and that of the planar $\mathcal{N}=4$ SYM one-loop spectral problem are contained in our formalism. What is more, our investigation of $\left|\Psi_{4,2}\right\rangle$ in the oscillator basis reveals singular contributions to $\Psi_{4,2}$ in spinor helicity variables that have been neglected so far.

\subsection{R-matrices of Heisenberg chain and spectral problem}

The evaluation of the unitary Graßmannian integral (5.7) for the $\mathfrak{u}(p, q \mid r+s)$ Yangian invariant $\left|\Psi_{4,2}\right\rangle$ parallels that in case of the bosonic algebra $\mathfrak{u}(p, q)$ published in [69]. To begin with, we parameterize the $\mathrm{U}(2)$ integration variable in (5.7) as

$$
\mathcal{C}=\left(\begin{array}{ll}
e^{i(\gamma+\alpha)} \cos \theta & -e^{i \beta} \sin \theta \\
e^{i(\gamma-\beta)} \sin \theta & e^{-i \alpha} \cos \theta
\end{array}\right)
$$


with $\alpha, \beta, \gamma \in[0,2 \pi]$ and $\theta \in\left[0, \frac{\pi}{2}\right]$. The Haar measure $[\mathrm{d} \mathcal{C}]=(2 \pi)^{-3} \sin (2 \theta) \mathrm{d} \theta \mathrm{d} \alpha \mathrm{d} \beta \mathrm{d} \gamma$ can then be obtained from (3.9). We rewrite the integrals in the variables $\alpha, \beta$, and $\gamma$ as complex contour integrals around unit circles and evaluate them using the residue theorem. The remaining integral in $\theta$ then reduces to an integral representation of the Euler beta function $\mathrm{B}(x, y)$. This leads to the invariant

$$
\begin{aligned}
\left|\Psi_{4,2}\right\rangle=\sum_{\substack{g_{13}, \ldots, g_{24}=0 \\
h_{13} \ldots, h_{24}=0 \\
\text { with }(6.3)}}^{\infty} & \frac{(1 \bullet 3)^{g_{13}}}{g_{13} !} \frac{(1 \bullet 4)^{h_{13}}}{g_{14} !} \frac{(1 \circ 4)^{h_{14}}}{h_{13} !} \frac{(2 \circ 3)^{g_{23}}}{h_{14} !} \frac{(2 \bullet 4)^{g_{24}}}{g_{24} !} \\
h_{23} ! & \frac{(2 \circ 4)^{h_{24}}}{h_{24} !}|0\rangle \\
& \cdot(-1)^{g_{14}+h_{14}} \mathrm{~B}\left(g_{14}+h_{23}+1, h_{13}+g_{24}-v_{1}+v_{2}\right) .
\end{aligned}
$$

In this formula the summation range is constrained by

$$
\begin{aligned}
& g_{13}-h_{13}+g_{14}-h_{14}=g_{13}-h_{13}+g_{23}-h_{23}=-c_{1}+q-s, \\
& g_{23}-h_{23}+g_{24}-h_{24}=g_{14}-h_{14}+g_{24}-h_{24}=-c_{2}+q-s .
\end{aligned}
$$

These constraints assure that the eigenvalues of $\overline{\mathbf{C}}^{1}, \overline{\mathbf{C}}^{2}, \mathbf{C}^{3}, \mathbf{C}^{4}$ acting on the invariant are, respectively, $c_{1}, c_{2},-c_{1},-c_{2}$. Furthermore, we have to assume $\operatorname{Re}\left(v_{1}-v_{2}\right)<0$ in order for the beta function integral to converge. In our final formula (6.2), we dropped a numerical prefactor.

The Yangian invariant $\left|\Psi_{4,2}\right\rangle$ is of key importance because its Yangian invariance condition (2.15) is equivalent to a Yang-Baxter equation. This was originally pointed out in [35] in the context of the deformed amplitude $\mathcal{A}_{4,2}^{\text {(def) }}$, which we encountered in the review section 2.3. Because the Yang-Baxter equation is at the core of the QISM, the equivalence is most naturally established using the Yangian invariance condition (2.16) in this language, see e.g. the discussion in [1]. As a consequence, $\left|\Psi_{4,2}\right\rangle$ can be identified with an R-matrix, and thus we represent it graphically as

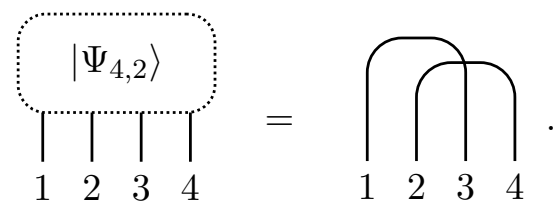

Let us fill in the details of this identification. The invariant $\left|\Psi_{4,2}\right\rangle$ from (6.2) is a state in $\overline{\mathscr{D}}_{c_{1}} \otimes \overline{\mathscr{D}}_{c_{2}} \otimes \mathscr{D}_{-c_{1}} \otimes \mathscr{D}_{-c_{2}}$. The representations $\overline{\mathscr{D}}_{c_{i}}$ and $\mathscr{D}_{-c_{i}}$ are dual to each other and therefore of the same dimension. Thus computing $\left|\Psi_{4,2}\right\rangle^{\dagger_{1} \dagger_{2}}$, where the conjugations act only on the respective oscillators, and then identifying the site indices $i, j$ of oscillators in the contractions $(i: j)$ as $1 \equiv 3$ and $2 \equiv 4$ results in an operator on $\mathscr{D}_{-c_{1}} \otimes \mathscr{D}_{-c_{2}}$. This is the R-matrix $R_{\mathscr{D}_{-c_{1}} \mathscr{D}_{-c_{2}}}(z)$ with the spectral parameter $z \equiv v_{1}-v_{2}$ and subscripts referring to $\mathfrak{u}(p, q \mid r+s)$ representations. Our Graßmannian integral (5.7) yields a novel $\mathrm{U}(2)$ integral formula for this R-matrix with oscillator representations.

It is instructive to execute this construction of the R-matrix in the simplest case conceivable, i.e. for the compact bosonic algebra $\mathfrak{u}(2,0) \cong \mathfrak{u}(2)$ with $c_{1}=c_{2}=-1$. Here 
the intricate sums in (6.2) reduce to just two terms,

$$
\left|\Psi_{4,2}\right\rangle=\frac{[z(1 \bullet 3)(2 \bullet 4)+(1 \bullet 4)(2 \bullet 3)]|0\rangle}{z(1-z)} .
$$

The corresponding R-matrix acts on two copies of the space $\mathscr{D}_{1}=\mathbb{C}^{2}$. Each copy is spanned by two states $\overline{\mathbf{a}}_{1}^{i}|0\rangle$ and $\overline{\mathbf{a}}_{2}^{i}|0\rangle$, which are built from the creation operators (5.2) contained in the oscillator contractions. These states are realized in the following as $\left(\begin{array}{l}1 \\ 0\end{array}\right)$ and $\left(\begin{array}{l}0 \\ 1\end{array}\right)$, respectively. This gives rise to

$$
R_{\mathscr{D}_{1} \mathscr{D}_{1}}(z)=\frac{1}{z(1-z)}\left(\begin{array}{cccc}
1+z & 0 & 0 & 0 \\
0 & z & 1 & 0 \\
0 & 1 & z & 0 \\
0 & 0 & 0 & 1+z
\end{array}\right)
$$

which is the R-matrix of the celebrated Heisenberg spin chain, as reviewed e.g. in [16]. This connection illustrates the relevance of the unitary Graßmannian integral approach for integrable spin chain models with their vast associated literature. Next, we return to the general setting with the non-compact superalgebra $\mathfrak{u}(p, q \mid r+s)$. A formula for the R-matrix corresponding to the Yangian invariant $\left|\Psi_{4,2}\right\rangle$ in (6.2) was already worked out in [35]. For the algebra $\mathfrak{u}(2,2 \mid 4)$ it is essentially ${ }^{9}$ the R-matrix of the spin chain governing the planar $\mathcal{N}=4 \mathrm{SYM}$ one-loop spectral problem [45, 46]. In view of this result, the Bargmann transformation from section 5.4 is an explicit change of basis from the oscillator R-matrix of the spectral problem to the Yangian invariant $\Psi_{4,2}$ in spinor helicity variables, which appears to be a deformation of the amplitude $\mathcal{A}_{4,2}$, recall section 4.1.

The $\mathrm{U}(2)$ integral formula for the $\mathfrak{u}(p, q \mid r+s)$ R-matrix obtained from the Graßmannian integral (5.7), as explained in this section, suggests several generalizations that we deem worthy to be explored. One might wonder whether the formula can be extended beyond the class of oscillator representations of section 5.1. Even further, it might exist on the algebraic level of the universal R-matrix, see e.g. [84] and the original references therein. This would be a path to elucidate a possible quantum group origin of the Graßmannian integral. A different direction would be to extend our U(2) integral formula to Beisert's R-matrix [47] with centrally extended $\mathfrak{s u}(2 \mid 2)$ symmetry. Two copies of this solution to the Yang-Baxter equation describe the asymptotic scattering of excitations in the all-loop spectral problem of planar $\mathcal{N}=4$ SYM. Moreover, it generalizes Shastry's R-matrix [85] for the one-dimensional Hubbard model. This line of research could be a stepping stone to a unitary Graßmannian integral for Yangian invariants associated with all-loop amplitudes.

\subsection{Normalization and divergent terms}

Here we inspect the normalization of the $\mathfrak{u}(p, q \mid r+s)$ Yangian invariant $\left|\Psi_{4,2}\right\rangle$ in (6.2), that arises naturally from the unitary Graßmannian integral (5.7) in oscillator variables. In the limit $z \equiv v_{1}-v_{2} \rightarrow 0$, we will observe a divergent $\frac{1}{z}$ contribution. In fact, we

\footnotetext{
${ }^{9}$ The oscillators in [46] satisfy non-standard reality conditions. This difference compared to our conventions in section 5.1, which follow [52,67], does not seem to affect the $\mathfrak{g l}(4 \mid 4)$ invariant R-matrix.
} 
recognize such a term immediately in the simple $\mathfrak{u}(2)$ case of $\left|\Psi_{4,2}\right\rangle$ displayed in (6.5), see also the formula (6.6) of the corresponding R-matrix. This observation seems to clash with the undeformed limit, $z \rightarrow 0$ and $c_{i}=0$, of $\Psi_{4,2}$ for $\mathfrak{u}(2,2 \mid 4)$ in spinor helicity variables discussed in section 4.1. There we did not encounter a divergent term but straightforwardly obtained the finite amplitude $\mathcal{A}_{4,2}$ in the limit. To resolve this apparent contradiction, we will diligently reexamine the evaluation of the Graßmannian integral (3.5) for $\Psi_{4,2}$. This will reveal a $\frac{1}{z}$ term that is only present for special kinematic configurations and is thereby easily overlooked. The existence of this term substantially affects the conceptual relation between the Yangian invariant $\Psi_{4,2}$ and the amplitude $\mathcal{A}_{4,2}$.

Let us consider $\left|\Psi_{4,2}\right\rangle$ for $\mathfrak{u}(p, q \mid r+s)$ with equal representation labels $c_{1}=c_{2}$, which is given in form of convoluted sums in (6.2). To extract the leading term as $z \rightarrow 0$, we expand the Euler beta functions in the summands. We find it to be a divergent $\frac{1}{z}$ contribution,

$$
\left|\Psi_{4,2}\right\rangle=\frac{1}{z}|\Upsilon\rangle_{14}|\Upsilon\rangle_{23}+\mathcal{O}\left(z^{0}\right) \quad \text { with } \quad|\Upsilon\rangle_{i j}=\sum_{\substack{g, h=0 \\ g-h=q-s-c_{i}}}^{\infty} \frac{(i \bullet j)^{g}}{g !} \frac{(i \circ j)^{h}}{h !}|0\rangle
$$

where we dropped an overall sign factor compared to (6.2). This result is conveniently expressed in terms of the state $|\Upsilon\rangle_{i j} \in \overline{\mathscr{D}}_{c_{i}} \otimes \mathscr{D}_{c_{j}=-c_{i}}$. Computing $|\Upsilon\rangle_{i j}^{\dagger_{i}}$ and then identifying the site indices of oscillators from the contractions $(i \bullet j)$ as $i \equiv j$ gives the identity operator on $\mathscr{D}_{-c_{i}}$, see [1]. Moreover, $|\Upsilon\rangle_{i j}$ is identical to the Yangian invariant $\left|\Psi_{2,1}\right\rangle$ defined by the Graßmannian integral (5.7) with some relabeling of the oscillators. Its expression as infinite sums in (6.7) matches the series expansion of a modified Bessel function of the first kind. This Bessel function is the simplest $1 \times 1$ case of the determinant formula for $\left|\Psi_{2 K, K}\right\rangle$ with the special Graßmannian integrand (5.11) mentioned towards the end of section 5.2. Eventually, we can infer from (6.7) the normalization of the R-matrix $R_{\mathscr{D}_{-c_{1}} \mathscr{D}_{-c_{1}}}(z)$, which is constructed from $\left|\Psi_{4,2}\right\rangle$ as described in section 6.1. Often an R-matrix is normalized such that, for vanishing spectral parameter $z=0$, it is finite and reduces to a permutation operator, see e.g. the textbook [86]. In contrast, $R_{\mathscr{D}_{-c_{1}} \mathscr{D}_{-c_{1}}}(z)$ diverges for $z \rightarrow 0$ as $\frac{1}{z}$, and the coefficient of this divergence is the permutation operator ${ }^{10}$ on $\mathscr{D}_{-c_{1}} \otimes \mathscr{D}_{-c_{1}}$. This can be observed very explicitly for the simple $\mathfrak{u}(2)$ R-matrix $R_{\mathscr{D}_{1} \mathscr{D}_{1}}(z)$ in (6.6), where the permutation operator on $\mathbb{C}^{2} \otimes \mathbb{C}^{2} \cong \mathbb{C}^{4}$ is just a $4 \times 4$ matrix. In conclusion, the unitary Graßmannian integral (5.7) for $\left|\Psi_{4,2}\right\rangle$ leads to an uncommon normalization of the corresponding R-matrix.

The unitary Graßmannian integrals (5.7) for $\left|\Psi_{4,2}\right\rangle$ in oscillator variables and (3.5) for $\Psi_{4,2}$ in spinor helicity variables are related by a change of basis, which is implemented by the Bargmann transformation of section 5.4. Therefore the $\frac{1}{z}$ term has also to be present for $\Psi_{4,2}$. Before extracting it from the Graßmannian integral (3.5), it is helpful to recapitulate a similar but simpler calculation. Also the gamma function $\Gamma(z)$ diverges for $z \rightarrow 0$ as $\frac{1}{z}$.

\footnotetext{
${ }^{10}$ The identification of oscillator indices below (6.4), which is needed for the R-matrix, differs from that mentioned after (6.7). Thus we obtain the permutation operator and not the identity.
} 
This can be shown using its Euler integral representation valid for $\operatorname{Re} z>0$, see e.g. [87],

$$
\Gamma(z)=\int_{0}^{\infty} \mathrm{d} \tau e^{-\tau} \tau^{z-1}=\frac{1}{z} \int_{0}^{\infty} \mathrm{d} \tau e^{-\tau} \frac{\mathrm{d}}{\mathrm{d} \tau} \tau^{z}=\frac{1}{z}+\mathcal{O}\left(z^{0}\right) .
$$

The first integrand can be expanded into non-negative powers of $z$ for $z \rightarrow 0$. However, because of the singularity at $\tau=0$, this expansion does not commute with the integration. Thus we rewrite the gamma function as the second integral in (6.8). After performing a partial integration, we are left with an integrand that is regular in $\tau$. Its expansion in $z$ can be integrated term by term and yields the rightmost side of (6.8).

We apply a similar strategy to the $\mathfrak{u}(2,2 \mid 0+4)$ Yangian invariant $\Psi_{4,2}$ with $c_{1}=c_{2}$ defined by the unitary Graßmannian integral (3.5). We parameterize the $\mathrm{U}(2)$ integration variable $\mathcal{C}$ as in (6.1). To extract the divergent term, we concentrate on the integral in $\theta$ because the integrand (3.13) of the Graßmannian formula has only singularities in this variable. Proceeding essentially along the lines of (6.8) for this integral and assuming ${ }^{11}$ $\operatorname{Re} z<0$ to eliminate certain boundary terms, we isolate the leading $\frac{1}{z}$ contribution. After performing the remaining trivial integrals in $\alpha, \beta$, and $\gamma$, we are left with

$$
\Psi_{4,2}=\frac{1}{z} \Upsilon_{14} \Upsilon_{23}+\mathcal{O}\left(z^{0}\right) \text { with } \Upsilon_{i j}=\left(\frac{\lambda_{1}^{i}}{\lambda_{1}^{j}}\right)^{c_{i}} \frac{\overline{\lambda_{1}^{i}}}{\left(\lambda_{1}^{j}\right)^{2} \lambda_{1}^{i}} \delta^{3}\left(p^{i}+p^{j}\right) \delta^{0 \mid 4}\left(\lambda_{1}^{i} \tilde{\eta}^{i}+\lambda_{1}^{j} \tilde{\eta}^{j}\right),
$$

where we neglected a numerical prefactor. The three-dimensional delta function $\delta^{3}(P)=$ $\delta\left(P_{11}\right) \delta_{\mathbb{C}}\left(P_{21}\right)$ suffices because it implies $P_{22}=0$ for $P$ being the sum of two massless momenta. Clearly, the decisive property of the divergent $\frac{1}{z}$ term in (6.9) is its absence for generic momentum configurations with $p^{1}+p^{2}+p^{3}+p^{4}=0$ as it only contributes for special kinematics with $p^{1}+p^{4}=0$ and $p^{2}+p^{3}=0$. Thereby it is easily overlooked in the spinor helicity basis.

What are the implications of the divergent term derived in the present section? The evaluation of the unitary Graßmannian integral (3.5) for the $\mathfrak{u}(2,2 \mid 0+4)$ Yangian invariant $\Psi_{4,2}$ in section 4.1 resulted in the expression (4.2). This reduces to the amplitude $\mathcal{A}_{4,2}$ in the undeformed limit $z \rightarrow 0$ and $c_{i}=0$. Our careful reexamination of the integral here revealed that (4.2) has to be supplemented by the $\frac{1}{z}$ term in (6.9). Thus, strictly speaking, the undeformed limit of $\Psi_{4,2}$ is divergent and $\mathcal{A}_{4,2}$ occurs as the coefficient of $z^{0}$ in an expansion for small $z$. This role of the amplitude is very similar to that in [43], to which we referred to already at the very end of the review section 2.3. In that reference, $\mathcal{A}_{4,2}$ is constructed from the one-loop dilatation generator of the planar $\mathcal{N}=4 \mathrm{SYM}$ spectral problem, rather than directly from the Yangian invariant R-matrix of the one-loop spin chain. This interpretation of the amplitude $\mathcal{A}_{4,2}$ does not mean that it is not "integrable" but only changes its conceptual place within the integrable structure.

What can we learn from the $\frac{1}{z}$ divergence of $\Psi_{4,2}$ about the general $\mathfrak{u}(2,2 \mid 0+4)$ Yangian invariant $\Psi_{2 K, K}$ defined by the unitary Graßmannian integral (3.5)? Iterating the recursion (B.1) and switching from the oscillator basis there to spinor helicity variables, we

\footnotetext{
${ }^{11}$ This is consistent as the same assumption was required to evaluate the oscillator invariant $\left|\Psi_{4,2}\right\rangle$ in (6.2).
} 
find that $\Psi_{2 K, K}$ can be constructed by gluing together $\frac{1}{2} K(K-1)$ copies of $\Psi_{4,2}$. Focusing on $\Psi_{2 K, K}$ with equal parameters $c_{i}$ and also equal $z_{i} \equiv v_{i}-v_{i+1}$ denoted $z$, the complex deformation parameter of each $\Psi_{4,2}$ turns out to be a multiple of $z$. Thus the leading term of $\Psi_{2 K, K}$ as $z \rightarrow 0$ is of order $z^{-\frac{1}{2} K(K-1)}$. In our crude evaluation of the Graßmannian formula for $\Psi_{6,3}$ in section 4.2 , we did not observe any divergent terms. Hence the terms of orders $\frac{1}{z^{3}}, \frac{1}{z^{2}}$, and $\frac{1}{z}$ have to be restricted to special kinematic configurations. Consequently, we could naively obtain the amplitude $\mathcal{A}_{6,3}$ in one kinematic region simply by taking the undeformed limit of $\Psi_{6,3}$. Our discussion of the Yangian invariant $\Psi_{8,4}$ in section 4.4 implies from the current vantage perspective that there exist terms of orders $\frac{1}{z^{6}}$ through $\frac{1}{z^{3}}$ for special kinematics. At $\frac{1}{z^{2}}$ and $\frac{1}{z}$ there are contributions for generic kinematics, which cause even a naive undeformed limit of $\Psi_{8,4}$ to diverge. Knowing about the divergent terms of $\Psi_{4,2}$ and $\Psi_{6,3}$ for special kinematic configurations makes the sudden appearance of divergencies for generic kinematics in case of $\Psi_{8,4}$ less surprising. Notwithstanding these new insights, it remains both a challenging and imperative task to identify the amplitude $\mathcal{A}_{8,4}$ in the small- $z$ expansion of the Yangian invariant $\Psi_{8,4}$.

\section{$7 \quad$ Link to cusp equation}

Until this point, we meticulously explored the mathematical consequences of the unitary Graßmannian integral formulas (3.5) and (5.7). To catch a glimpse of where this research might be headed, we proceed less stringently in this section. This will reveal an intriguing connection to the equation $[76,77]$ that is believed to govern the so-called cusp anomalous dimension in planar $\mathcal{N}=4 \mathrm{SYM}$. This function of the 't Hooft coupling can be extracted from the anomalous dimensions of leading-twist operators. It is an integral part of the conjectured all-loop expression for MHV amplitudes [88], which is known to receive corrections starting at six particles [89]. Therefore our systematic approach to Yangian invariants appears to contain already hints of all-loop results.

To expose them, we consider the Yangian invariant $\left|\Psi_{4,2}\right\rangle$ in the oscillator basis given by the Graßmannian integral (5.7) with a U(2) contour. Specializing the deformation parameters $v_{i}^{ \pm}$such that its integrand $\mathscr{F}(\mathcal{C})$ is of the form $(5.11)$ reduces the integral to the Leutwyler-Smilga model [70]. Ordinarily, this integral for $\left|\Psi_{4,2}\right\rangle$ contains the $2 \times 2$ matrices Io, whose entries are built out of oscillator variables associated with $\mathfrak{u}(p, q \mid m)$ representations, and a Fock vacuum $|0\rangle$. In what follows, we neglect this oscillator structure ad hoc, and we even impose constraints on the now numerical entries of the matrices $\mathbf{I}_{8}$. This results in a family of integrals indexed by an integer parameter,

$$
\hat{K}_{\nu}\left(t, t^{\prime}\right)=\frac{1}{2} \int_{\mathrm{U}(2)}[\mathrm{d} \mathcal{C}](\operatorname{det} \mathcal{C})^{\nu} e^{\operatorname{tr}\left(\mathbf{I}_{\bullet} \mathcal{C}^{\dagger}+\mathcal{C} \mathbf{I}_{\circ}^{t}\right)} \quad \text { with } \quad \mathbf{I}_{\bullet}=-\mathbf{I}_{\circ}=\frac{1}{2}\left(\begin{array}{cc}
t & 0 \\
0 & t^{\prime}
\end{array}\right) .
$$

These integrals can be computed with the determinant formula [74] mentioned previously in section 5.2. In particular,

$$
\hat{K}_{0}\left(t, t^{\prime}\right)=\frac{t J_{1}(t) J_{0}\left(t^{\prime}\right)-t^{\prime} J_{0}(t) J_{1}\left(t^{\prime}\right)}{t^{2}-t^{\prime 2}}, \quad \hat{K}_{1}\left(t, t^{\prime}\right)=\frac{t^{\prime} J_{1}(t) J_{0}\left(t^{\prime}\right)-t J_{0}(t) J_{1}\left(t^{\prime}\right)}{t^{2}-t^{\prime 2}},
$$

where $J_{\nu}(t)$ denotes the standard Bessel function. 
Excitingly, precisely these two functions make up the kernel of the cusp equation in the planar $\mathcal{N}=4$ theory. The cusp anomalous dimension $f(g)$ depends on the 't Hooft coupling constant $\lambda$ via $g^{2}=\frac{\lambda}{16 \pi^{2}}$. It is given as $f(g)=16 g^{2} \hat{\sigma}(0)$ with the fluctuation density $\hat{\sigma}(t)$ obeying the integral equation $[76,77]$

$$
\hat{\sigma}(t)=\frac{t}{e^{t}-1}\left[\hat{K}(2 g t, 0)-4 g^{2} \int_{0}^{\infty} \mathrm{d} t^{\prime} \hat{K}\left(2 g t, 2 g t^{\prime}\right) \hat{\sigma}\left(t^{\prime}\right)\right] .
$$

The complete kernel for this equation, which incorporates effects from the asymptotic Bethe ansatz and the dressing phase, was found in [77],

$$
\hat{K}\left(t, t^{\prime}\right)=\hat{K}_{0}\left(t, t^{\prime}\right)+\hat{K}_{1}\left(t, t^{\prime}\right)+8 g^{2} \int_{0}^{\infty} \mathrm{d} t^{\prime \prime} \hat{K}_{1}\left(t, 2 g t^{\prime \prime}\right) \frac{t^{\prime \prime}}{e^{t^{\prime \prime}}-1} \hat{K}_{0}\left(2 g t^{\prime \prime}, t^{\prime}\right) .
$$

Thus we established a link from the $\mathrm{U}(2)$ integral expression (5.7) for the Yangian invariant $\left|\Psi_{4,2}\right\rangle$ to the building blocks (7.2) of the cusp kernel. It is on a heuristic level at present. Presumably, details of the $\mathfrak{u}(2,2 \mid 4)$ representations have to be injected to overcome, for instance, the ad hoc choice of the matrices in (7.1). Still, we are intrigued by these seeds of all-loop results in our unitary Graßmannian integral approach to the construction of tree-level amplitudes.

\section{Conclusions and outlook}

We began this article by recalling aspects of our and others' earlier work on the systematic construction of Yangian invariants from integrability in section 2. This allows, in contradistinction to just "observing" it, to take Yangian symmetry as a starting point, to then employ the quantum inverse scattering method, and to finally systematically construct such invariants. Applying this methodology in section 3 for the general symmetry algebra $\mathfrak{u}(p, p \mid m)$, while paying close attention to its correct reality conditions, we end up, in the special case of $N=2 K$ points and superhelicity $K$, with a family of unitary Graßmannian "contour" integrals. The construction requires a certain deformation of the naive integrands by extra parameters.

Evaluating the deformed integrals in case of the superconformal algebra $\mathfrak{p s u}(2,2 \mid 4)$ for $N=4,6,8$ points, attempting to remove the deformation parameters, and comparing to the corresponding, known, physical tree-level amplitudes, one, surprisingly, finds differences that become more and more pronounced as $N$ increases, cf. section 4 . For $N=4$ at generic momenta one reproduces the four-particle MHV amplitude. However, even here a closer look unveils the existence of certain contact terms at collinear momenta that diverge upon taking the deformation parameters to zero, see section 6.2 and in particular (6.9). For $N=6$ at generic momenta the physical six-particle NMHV amplitude is only reproduced in one out of four kinematical sectors, while terms divergent in the deformation parameters again appear at various collinear momentum configurations. An analogous toy integral for the case $N=4$ and the algebra $\mathfrak{u}(1,1)$ reproduces the proper R-matrix of this simpler 
algebra. This forces us to conclude that the physical six-particle NMHV amplitude in $\mathcal{N}=4 \mathrm{SYM}$ is not "as integrable as hoped for". These tensions between the Yangian invariant and the physical amplitude worsen for $N=8$. Divergences in the deformation parameters are now present even at generic momenta. Furthermore, the residues of these terms apparently no longer combine to the physical eight-point $\mathrm{N}^{2} \mathrm{MHV}$ amplitude in any kinematical sector [65].

What does this mean, especially in the light of the fact that there have been local proofs of Yangian invariance of the physical amplitudes in the planar $\mathcal{N}=4$ model, using differential operators representing the algebra? We suspect that Yangian invariance of the perturbative physical amplitudes is nevertheless subtly broken. This includes, but significantly goes beyond the effects already observed in [22-24]. One explanation would be that the physical amplitudes are only infinitesimally invariant, and that Yangian invariance (meaning either conformal or dual-conformal symmetry, or both) is broken by large transformations. We are currently investigating this possibility [58]. On the positive side, we found some evidence for Yangian invariance at all-loop level, cf. section 7. Could it be that the detected problems with the Yangian invariance of the tree-level scattering amplitudes of $\mathcal{N}=4 \mathrm{SYM}$ may be elegantly resolved at the non-perturbative, all-loop level?

\section{Acknowledgments}

We thank Jacob Bourjaily and Gregor Richter for earlier collaboration and help on several questions related to this work. We are thankful to Lance Dixon, Harald Dorn, Livia Ferro, Andrew Hodges, and Lionel Mason for valuable discussions. This work was funded in part by the Deutsche Forschungsgemeinschaft (DFG, German Research Foundation) under project number 270039613 .

\section{A Parity symmetry}

In this appendix, we investigate a discrete symmetry transformation of the unitary Graßmannian integrals (3.5) for $\Psi_{2 K, K}$ in spinor helicity variables and (5.7) for $\left|\Psi_{2 K, K}\right\rangle$ in oscillator variables. We define this so-called parity transformation $\mathcal{P}$ by reversing the order of the particles $i=1, \ldots, K$ with negative energies and also that of $i=K+1, \ldots, 2 K$ with positive energies. On the level of the two Graßmannian integral formulas, this transformation acts as, respectively,

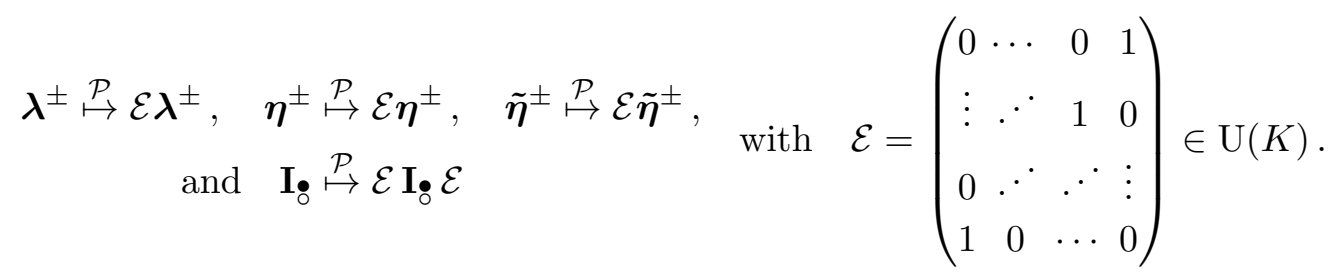

Here $\boldsymbol{\eta}^{ \pm}$are $K \times r$ blocks and $\tilde{\boldsymbol{\eta}}^{ \pm}$are $K \times s$ blocks of fermionic variables, which are defined analogously to the blocks $\boldsymbol{\lambda}^{ \pm}$of $\boldsymbol{\lambda}$ in (3.1). The transformation $\mathcal{P}$ is a symmetry of both 
Graßmannian integral formulas if the deformation parameters satisfy

$$
v_{1}-v_{2}=v_{2}-v_{3}=\cdots=v_{K-1}-v_{K}, \quad c_{1}=c_{2}=\cdots=c_{K} .
$$

This can be proven using the left- and right-invariance of Haar measure and the identity $[1,2, \ldots, j]_{\mathcal{E C E}}=\overline{[1,2, \ldots, K-j]_{\mathcal{C}}} \operatorname{det} \mathcal{C}$ for the minors appearing in the manifestly singlevalued Graßmannian integrand in (3.13).

We focus on the action of $\mathcal{P}$ on the Yangian invariant $\left|\Psi_{4,2}\right\rangle$ given by (5.7). In this case (A.2) yields no constraints for the parameters $v_{1}, v_{2} \in \mathbb{C}$ and imposes equal representation labels $c_{1}=c_{2}$. In section 6 , we argued that $\left|\Psi_{4,2}\right\rangle$ can be understood as an R-matrix acting on the tensor product $\mathscr{D}_{-c_{1}} \otimes \mathscr{D}_{-c_{1}}$ of two $\mathfrak{u}(p, q \mid r+s)$ representations. On this level, $\mathcal{P}$ permutes the two tensor factors, which is a symmetry of this R-matrix. In the literature on integrable models, see e.g. [86], this property is known as "parity invariance", hence our name for $\mathcal{P}$.

\section{B Unitary contour from gluing}

We argued in section 3.1 that momentum conservation (3.4) naturally suggests to equip the Graßmannian integral (3.5), which computes the Yangian invariant $\Psi_{2 K, K}$, with a $\mathrm{U}(K)$ contour for the integration variable $\mathcal{C}$. Strictly speaking, however, it does not completely fix the contour. To illustrate this point, we consider the matrix $\mathcal{C}(\mathcal{U})$ in $(4.3)$ from the six-particle sample invariant $\Psi_{6,3}$ of $\mathfrak{u}(2,2 \mid 0+4)$. It obeys the momentum condition (3.4) for any $\mathcal{U} \in \mathbb{C}$ but is unitary only for $\mathcal{U} \in \mathrm{U}(1)$. Here, working in the oscillator basis, we derive the $\mathrm{U}(K)$ contour of the Graßmannian integral (5.7) for the $\mathfrak{u}(p, q \mid r+s)$ Yangian invariant $\left|\Psi_{2 K, K}\right\rangle$ by "gluing" together multiple copies of $\left|\Psi_{4,2}\right\rangle$ with $\mathrm{U}(2)$ contours. There is no reason to question the contour of $\left|\Psi_{4,2}\right\rangle$ because it leads to the correct R-matrix, as explained in section 6.1. Let us remark that our gluing procedure gives rise to a parameterization of $\mathrm{U}(K)$ in terms of $\mathrm{U}(2)$ matrices that can be traced back to the classic work [90].

We proceed by induction with the assumption that $\left|\Psi_{2 K, K}\right\rangle$ is obtained from a Graßmannian integral with $\mathrm{U}(K)$ contour. We glue onto it invariants $\left|\Psi_{4,2}^{(t)}\right\rangle$ with $t=1, \ldots, K$ and show that the result yields a Graßmannian integral formula for $\left|\Psi_{2(K+1), K+1}\right\rangle$ with a $\mathrm{U}(K+1)$ contour. This is best described graphically,

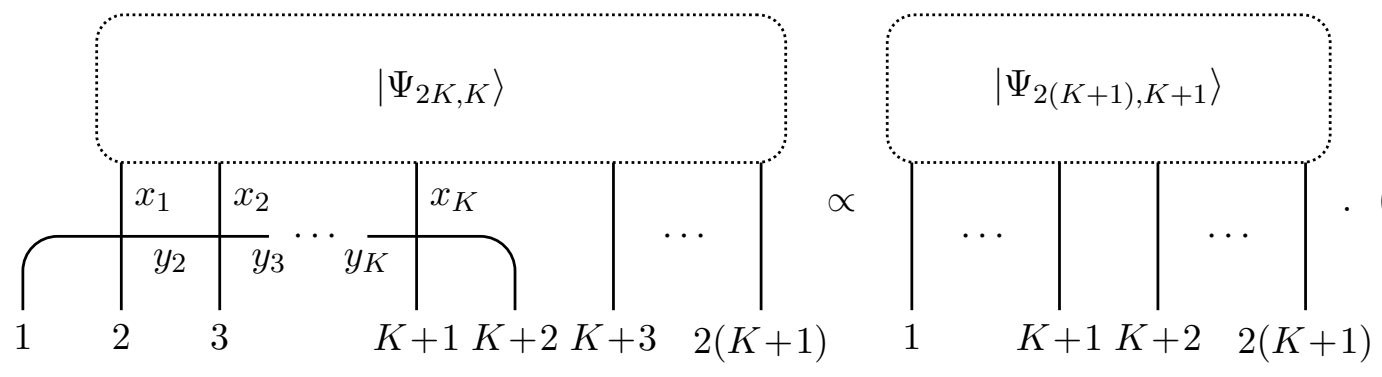

Here we visualized the $\left|\Psi_{4,2}^{(t)}\right\rangle$ as in (6.4) by intersections of lines. Moreover, the labels $x_{i}$ and $y_{i}$ are oscillator site indices associated with internal lines. Translated into a formula, (B.1) 
becomes

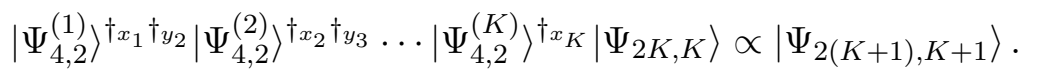

On the left-hand side, the invariants are obtained from the unitary Graßmannian integral formula (5.7) with some relabeling of the oscillator contractions (5.8) and the deformation parameters in the integrands (3.13) to be in accordance with (B.1). This reads for the invariants $\left|\Psi_{2 K, K}\right\rangle$ and $\left|\Psi_{4,2}^{(t)}\right\rangle$, respectively,

$$
\begin{aligned}
& (i \bullet j) \mapsto\left(x_{i}: j+2\right), \quad\left(v_{i}, c_{i}\right) \mapsto\left(v_{i+1}, c_{i+1}\right) \quad \text { for } \quad \begin{array}{l}
i=1, \ldots, K, \\
j=K+1, \ldots, 2 K,
\end{array} \\
& \left(\begin{array}{cc}
(1: 3) & (1: 4) \\
(2: 3) & (2: 4)
\end{array}\right) \mapsto\left(\begin{array}{cc}
\left(y_{t}: y_{t+1}\right) & \left(y_{t}: x_{t}\right) \\
\left(t+1: y_{t+1}\right) & \left(t+1: x_{t}\right)
\end{array}\right), \quad \begin{array}{l}
\left(v_{1}, c_{1}\right) \quad \text { unchanged } \\
\left(v_{2}, c_{2}\right) \mapsto\left(v_{t+1}, c_{t+1}\right)
\end{array}
\end{aligned}
$$

with $y_{1} \equiv 1$ and $y_{K+1} \equiv K+2$. The right-hand side of (B.2) is given by the Graßmannian formula (5.7) without any replacements. In what follows, we prove that both sides are indeed proportional.

We begin by manipulating the left-hand side of (B.2). The oscillators with site indices $x_{i}$ and $y_{i}$ appear only inside of vacuum expectation values because they are associated with internal lines in (B.1). We eliminate them by calculating these expectation values. What is more, we combine the integrands of the $\mathrm{U}(2)$ integrals and the $\mathrm{U}(K)$ integral. This yields

$$
\int_{\mathrm{U}(2)}\left[\mathrm{d} \mathcal{D}^{(1)}\right] \cdots \int_{\mathrm{U}(2)}\left[\mathrm{d} \mathcal{D}^{(K)}\right] \int_{\mathrm{U}(K)}[\mathrm{d} \mathcal{D}]\left|\mathcal{D}_{13}^{(2)}\right|^{2} \cdots\left|\mathcal{D}_{13}^{(K)}\right|^{2(K-1)} \mathscr{F}(\mathcal{C})(\operatorname{det} \mathcal{C})^{r} e^{\operatorname{tr}\left(\mathbf{I}_{\bullet} \mathcal{C}^{\dagger}+\mathcal{C} \mathbf{I}_{\circ}^{t}\right)}|0\rangle,
$$

where we denote the integration variables originating from $\left|\Psi_{2 K, K}\right\rangle$ and $\left|\Psi_{4,2}^{(t)}\right\rangle$ by $\mathcal{D}$ and $\mathcal{D}^{(t)}$, respectively. The integrand $\mathscr{F}(\mathcal{C})$ and the exponential with the $\mathrm{U}(K+1)$ matrix

$$
\mathcal{C}=\left(\begin{array}{cc:c}
\mathcal{D}_{13}^{(1)} & \mathcal{D}_{14}^{(1)} & 0 \\
\mathcal{D}_{23}^{(1)} & \mathcal{D}_{24}^{(1)} & 0 \\
\hdashline 0 & 1_{K-1}
\end{array}\right) \cdots\left(\begin{array}{c:c:c}
\mathcal{D}_{13}^{(K)} & 0 & \mathcal{D}_{14}^{(K)} \\
\hdashline 0 & 1_{K-1} & 0 \\
\hdashline \mathcal{D}_{23}^{(K)} & 0 & \mathcal{D}_{24}^{(K)}
\end{array}\right)\left(\begin{array}{c:c}
1 & 0 \\
\hdashline 0 & \mathcal{D}
\end{array}\right)
$$

are already those of $\left|\Psi_{2(K+1), K+1}\right\rangle$. Therefore we can focus on the Haar measures in (B.4) next. Starting out a bit more general, we introduce the complex Stiefel manifold $V_{L}\left(\mathbb{C}^{M}\right)$ with $L \leq M$ which is the set of $M \times L$ matrices $\mathcal{S}$ satisfying $\mathcal{S}^{\dagger} \mathcal{S}=1_{L}$. It generalizes the unitary group manifold to non-square matrices as $V_{M}\left(\mathbb{C}^{M}\right)=\mathrm{U}(M)$. Its Haar measure can be realized as

$$
\int_{V_{L}\left(\mathbb{C}^{M}\right)}[\mathrm{d} \mathcal{S}] \propto \int_{\mathbb{C}^{L M}} \frac{\mathrm{d}^{L M} \mathcal{S}^{\dagger} \mathrm{d}^{L M} \mathcal{S}}{(2 i)^{L M}} \delta^{L^{2}}\left(\mathcal{S}^{\dagger} \mathcal{S}-1_{L}\right)
$$

where the delta function of a Hermitian matrix is defined as the product of real delta functions of its diagonal elements times complex delta functions of the upper triangular ones. We implement the Haar measures $\left[\mathrm{d} \mathcal{D}^{(t)}\right]$ in (B.4) as in (B.6) and eliminate degrees 
of freedom using the delta functions. The factors $\left|\mathcal{D}_{13}^{(t)}\right|^{2}$ in (B.4) cancel with factors arising from these measures. Further degrees of freedom can be integrated out because the matrices $\mathcal{D}^{(t)}$ appear only in one particular combination inside of $\mathcal{C}$ in (B.5). Yet others can be absorbed into $\mathcal{D}$ using the left-invariance of $[\mathrm{d} \mathcal{D}]$. As a result, (B.4) becomes

$$
\int_{V_{1}\left(\mathbb{C}^{K+1}\right)}[\mathrm{d} \mathcal{S}] \int_{\mathrm{U}(K)}[\mathrm{d} \mathcal{D}] \mathscr{F}(\mathcal{C})(\operatorname{det} \mathcal{C})^{r} e^{\operatorname{tr}\left(\mathbf{I}_{\bullet} \mathcal{C}^{\dagger}+\mathcal{C} \mathbf{I}_{\circ}^{t}\right)}|0\rangle,
$$

where we neglected a numerical prefactor, and we have

$$
\mathcal{C}=\left(\begin{array}{c:c}
\mathcal{S} & \mathcal{T}(\mathcal{S})
\end{array}\right)\left(\begin{array}{c:c}
1 & 0 \\
\hdashline 0 & \mathcal{D}
\end{array}\right) \in \mathrm{U}(K+1) .
$$

The first factor in $\mathcal{C}$ stems from multiplying the first $K$ factors in (B.5), and the $K+1 \times K$ matrix $\mathcal{T}(\mathcal{S})$ is completely determined in terms of $\mathcal{S}$. The Stiefel manifold in (B.7) is just a unit sphere and can also be interpreted as a coset space, $V_{1}\left(\mathbb{C}^{K+1}\right) \cong S^{2 K+3} \cong$ $\mathrm{U}(K+1) / \mathrm{U}(K)$. The first factor in (B.8) is a representative of the coset space element $\mathcal{S}$ in $\mathrm{U}(K+1)$. With this interpretation, the product of the two integrals in (B.7) equals $\int_{\mathrm{U}(K+1)}[\mathrm{d} \mathcal{C}]$, see e.g. the explanation around equation (5.121) in [91]. Therefore (B.7) is nothing but the right-hand side of (B.2). Q.E.D.

Open Access. This article is distributed under the terms of the Creative Commons Attribution License (CC-BY 4.0), which permits any use, distribution and reproduction in any medium, provided the original author(s) and source are credited.

\section{References}

[1] N. Kanning, On the integrable structure of super Yang-Mills scattering amplitudes, Ph.D. Thesis, Humboldt University, Berlin Germany (2016) [arXiv:1811.06324] [INSPIRE].

[2] L.J. Dixon, A brief introduction to modern amplitude methods, in Proceedings of 2012 European School of High-Energy Physics (ESHEP 2012), La Pommeraye France (2012), pg. 31 [arXiv:1310.5353] [INSPIRE].

[3] J.M. Henn and J.C. Plefka, Scattering Amplitudes in Gauge Theories, Lect. Notes Phys. 883 (2014) 1 .

[4] H. Elvang and Y.-t. Huang, Scattering Amplitudes in Gauge Theory and Gravity, Cambridge University Press, Cambridge U.K. (2015).

[5] R. Roiban, Review of AdS/CFT Integrability, Chapter V.1: Scattering Amplitudes - a Brief Introduction, Lett. Math. Phys. 99 (2012) 455 [arXiv:1012.4001] [InSPIRE].

[6] J.M. Drummond, Review of AdS/CFT Integrability, Chapter V.2: Dual Superconformal Symmetry, Lett. Math. Phys. 99 (2012) 481 [arXiv:1012.4002] [INSPIRE].

[7] R. Kleiss and H. Kuijf, Multi-gluon cross-sections and five jet production at hadron colliders, Nucl. Phys. B 312 (1989) 616 [InSPIRE].

[8] B.L. van der Waerden, Spinoranalyse, Gesell. Wiss. Göttingen Nachr. Math-Phys. Kl. 1929 (1928) 100, http://eudml.org/doc/59283. 
[9] H. Weyl, Electron and Gravitation. 1 (in German), Z. Phys. 56 (1929) 330 [inSPIRE].

[10] J.M. Drummond and J.M. Henn, All tree-level amplitudes in N=4 SYM, JHEP 04 (2009) 018 [arXiv: 0808.2475] [INSPIRE].

[11] S.J. Parke and T.R. Taylor, An Amplitude for $n$ Gluon Scattering, Phys. Rev. Lett. 56 (1986) 2459 [INSPIRE].

[12] V.P. Nair, A Current Algebra for Some Gauge Theory Amplitudes, Phys. Lett. B 214 (1988) 215 [INSPIRE].

[13] V.G. Drinfeld, Hopf algebras and the quantum Yang-Baxter equation, Sov. Math. Dokl. 32 (1985) 254 [INSPIRE].

[14] M.L. Nazarov, Quantum Berezinian and the Classical Capelli Identity, Lett. Math. Phys. 21 (1991) 123.

[15] F. Loebbert, Lectures on Yangian Symmetry, J. Phys. A 49 (2016) 323002 [arXiv: 1606. 02947] [INSPIRE].

[16] L.D. Faddeev, How algebraic Bethe ansatz works for integrable model, in Relativistic gravitation and gravitational radiation. Proceedings of School of Physics, Les Houches France (1995), pg. 149 [hep-th/9605187] [INSPIRE].

[17] R. Frassek, N. Kanning, Y. Ko and M. Staudacher, Bethe Ansatz for Yangian Invariants: Towards Super Yang-Mills Scattering Amplitudes, Nucl. Phys. B 883 (2014) 373 [arXiv: 1312.1693] [INSPIRE].

[18] D. Chicherin, S. Derkachov and R. Kirschner, Yang-Baxter operators and scattering amplitudes in $N=4$ super-Yang-Mills theory, Nucl. Phys. B 881 (2014) 467 [arXiv: 1309.5748] [INSPIRE].

[19] J.M. Drummond, J.M. Henn and J. Plefka, Yangian symmetry of scattering amplitudes in $N=4$ super Yang-Mills theory, JHEP 05 (2009) 046 [arXiv:0902.2987] [INSPIRE].

[20] N. Beisert, On Yangian Symmetry in Planar N=4SYM, in Quantum chromodynamics and beyond: Gribov-80 memorial volume. Proceedings of Memorial Workshop devoted to the 80th birthday of V.N. Gribov, Trieste Italy (2010), pg. 175 [arXiv:1004.5423] [INSPIRE].

[21] L. Ferro, J. Plefka and M. Staudacher, Yangian Symmetry in Maximally Supersymmetric Yang-Mills Theory, in Space-Time-Matter: Analytic and Geometric Structures, de Gruyter, Berlin Germany (2018), pg. 288.

[22] T. Bargheer, N. Beisert, W. Galleas, F. Loebbert and T. McLoughlin, Exacting $N=4$ Superconformal Symmetry, JHEP 11 (2009) 056 [arXiv:0905.3738] [INSPIRE].

[23] A. Sever and P. Vieira, Symmetries of the $N=4$ SYM S-matrix, arXiv:0908.2437 [INSPIRE].

[24] T. Bargheer, N. Beisert and F. Loebbert, Exact Superconformal and Yangian Symmetry of Scattering Amplitudes, J. Phys. A 44 (2011) 454012 [arXiv:1104.0700] [InSPIRE].

[25] N. Arkani-Hamed, F. Cachazo, C. Cheung and J. Kaplan, A Duality For The S Matrix, JHEP 03 (2010) 020 [arXiv:0907.5418] [InSPIRE].

[26] L.J. Mason and D. Skinner, Dual Superconformal Invariance, Momentum Twistors and Grassmannians, JHEP 11 (2009) 045 [arXiv:0909.0250] [InSPIRE].

[27] N. Arkani-Hamed, J.L. Bourjaily, F. Cachazo, A.B. Goncharov, A. Postnikov and J. Trnka, Scattering Amplitudes and the Positive Grassmannian, [arXiv:1212.5605] [InSPIRE]. 
[28] N. Arkani-Hamed, J. Bourjaily, F. Cachazo, A. Goncharov, A. Postnikov and J. Trnka, Grassmannian Geometry of Scattering Amplitudes, Cambridge University Press, Cambridge U.K. (2016).

[29] J.M. Drummond and L. Ferro, Yangians, Grassmannians and T-duality, JHEP 07 (2010) 027 [arXiv: 1001.3348] [INSPIRE].

[30] J.M. Drummond and L. Ferro, The Yangian origin of the Grassmannian integral, JHEP 12 (2010) 010 [arXiv: 1002.4622] [INSPIRE].

[31] J.L. Bourjaily, J. Trnka, A. Volovich and C. Wen, The Grassmannian and the Twistor String: Connecting All Trees in $N=4$ SYM, JHEP 01 (2011) 038 [arXiv:1006.1899] [INSPIRE].

[32] D. Nandan, A. Volovich and C. Wen, A Grassmannian Etude in NMHV Minors, JHEP 07 (2010) 061 [arXiv:0912.3705] [INSPIRE].

[33] N. Arkani-Hamed, J. Bourjaily, F. Cachazo and J. Trnka, Unification of Residues and Grassmannian Dualities, JHEP 01 (2011) 049 [arXiv:0912.4912] [INSPIRE].

[34] L. Ferro, T. Lukowski, C. Meneghelli, J. Plefka and M. Staudacher, Harmonic R-matrices for Scattering Amplitudes and Spectral Regularization, Phys. Rev. Lett. 110 (2013) 121602 [arXiv: 1212.0850] [INSPIRE].

[35] L. Ferro, T. Łukowski, C. Meneghelli, J. Plefka and M. Staudacher, Spectral Parameters for Scattering Amplitudes in N=4 Super Yang-Mills Theory, JHEP 01 (2014) 094 [arXiv: 1308.3494] [INSPIRE].

[36] N. Beisert, J. Broedel and M. Rosso, On Yangian-invariant regularization of deformed on-shell diagrams in $\mathcal{N}=4$ super-Yang-Mills theory, J. Phys. A 47 (2014) 365402 [arXiv: 1401.7274] [INSPIRE].

[37] J. Broedel, M. de Leeuw and M. Rosso, A dictionary between R-operators, on-shell graphs and Yangian algebras, JHEP 06 (2014) 170 [arXiv:1403.3670] [INSPIRE].

[38] J. Broedel, M. de Leeuw and M. Rosso, Deformed one-loop amplitudes in $\mathcal{N}=4$ super-Yang-Mills theory, JHEP 11 (2014) 091 [arXiv:1406.4024] [INSPIRE].

[39] L. Ferro, T. Lukowski and M. Staudacher, $\mathcal{N}=4$ scattering amplitudes and the deformed Graßmannian, Nucl. Phys. B 889 (2014) 192 [arXiv:1407.6736] [InSPIRE].

[40] T. Bargheer, Y.-t. Huang, F. Loebbert and M. Yamazaki, Integrable Amplitude Deformations for $N=4$ Super Yang-Mills and ABJM Theory, Phys. Rev. D 91 (2015) 026004 [arXiv: 1407.4449] [INSPIRE].

[41] D. Chicherin and R. Kirschner, Yangian symmetric correlators, Nucl. Phys. B 877 (2013) 484 [arXiv: 1306.0711] [INSPIRE].

[42] N. Kanning, T. Lukowski and M. Staudacher, A shortcut to general tree-level scattering amplitudes in $\mathcal{N}=4$ SYM via integrability, Fortsch. Phys. 62 (2014) 556 [arXiv:1403.3382] [INSPIRE].

[43] B.I. Zwiebel, From Scattering Amplitudes to the Dilatation Generator in $N=4 S Y M, J$. Phys. A 45 (2012) 115401 [arXiv: 1111.0083] [INSPIRE].

[44] N. Arkani-Hamed and J. Trnka, The Amplituhedron, JHEP 10 (2014) 030 [arXiv: 1312.2007] [INSPIRE].

[45] N. Beisert and M. Staudacher, The N=4 SYM integrable super spin chain, Nucl. Phys. B 670 (2003) 439 [hep-th/0307042] [INSPIRE]. 
[46] N. Beisert, The complete one loop dilatation operator of $N=4$ superYang-Mills theory, Nucl. Phys. B 676 (2004) 3 [hep-th/0307015] [INSPIRE].

[47] N. Beisert, The Analytic Bethe Ansatz for a Chain with Centrally Extended su(2|2) Symmetry, J. Stat. Mech. 0701 (2007) P01017 [nlin/0610017].

[48] S. Salaff, A Nonzero Determinant Related to Schur's Matrix, Trans. Am. Math. Soc. 127 (1967) 349.

[49] D.T. Stoyanov and I.T. Todorov, Majorana representations of the Lorentz group and infinite component fields, J. Math. Phys. 9 (1968) 2146 [InSPIRE].

[50] G. Mack and I. Todorov, Irreducibility of the ladder representations of u(2,2) when restricted to the Poincaré subgroup, J. Math. Phys. 10 (1969) 2078 [INSPIRE].

[51] I.T. Todorov, Discrete Series of Hermitian Representations of the Lie Algebra of $u(p, q)$, Preprint IC-66-71 (1966).

[52] I. Bars and M. Günaydin, Unitary Representations of Noncompact Supergroups, Commun. Math. Phys. 91 (1983) 31 [INSPIRE].

[53] N. Arkani-Hamed, F. Cachazo and C. Cheung, The Grassmannian Origin of Dual Superconformal Invariance, JHEP 03 (2010) 036 [arXiv:0909.0483] [INSPIRE].

[54] J.M. Drummond, J. Henn, G.P. Korchemsky and E. Sokatchev, Generalized unitarity for $N=4$ super-amplitudes, Nucl. Phys. B 869 (2013) 452 [arXiv:0808.0491] [inSPIRE].

[55] J.M. Drummond, J. Henn, G.P. Korchemsky and E. Sokatchev, Dual superconformal symmetry of scattering amplitudes in $N=4$ super-Yang-Mills theory, Nucl. Phys. B 828 (2010) 317 [arXiv:0807.1095] [INSPIRE].

[56] H. Dorn, H. Münkler and C. Spielvogel, Conformal geometry of null hexagons for Wilson loops and scattering amplitudes, Phys. Part. Nucl. 45 (2014) 692 [arXiv:1211.5537] [INSPIRE].

[57] A. Hodges, Eliminating spurious poles from gauge-theoretic amplitudes, JHEP 05 (2013) 135 [arXiv:0905.1473] [INSPIRE].

[58] N. Kanning and M. Staudacher, work in progress.

[59] K.I. Gross and R.A. Kunze, Fourier Bessel Transforms and Holomorphic Discrete Series, in Conference on Harmonic Analysis, College Park Maryland U.S.A. (1971), pg. 79.

[60] G. Post, Properties of Massless Relativistic Fields Under the Conformal Group, J. Math. Phys. 17 (1976) 24 [InSPIRE].

[61] G.P. Korchemsky and E. Sokatchev, Twistor transform of all tree amplitudes in $N=4$ SYM theory, Nucl. Phys. B 829 (2010) 478 [arXiv:0907.4107] [InSPIRE].

[62] L. Hua, Harmonic Analysis of Functions of Several Complex Variables in the Classical Domains, American Mathematical Society, Providence U.S.A. (1963).

[63] J.L. Bourjaily, Efficient Tree-Amplitudes in $N=4$ : Automatic BCFW Recursion in Mathematica, arXiv: 1011.2447 [INSPIRE].

[64] J.L. Bourjaily, Positroids, Plabic Graphs and Scattering Amplitudes in Mathematica, arXiv:1212.6974 [INSPIRE].

[65] N. Kanning and M. Staudacher, unpublished. 
[66] B.V. Shabat, Introduction to Complex Analysis: Part II, Functions of Several Variables, American Mathematical Society, Providence U.S.A. (1992).

[67] M. Günaydin and N. Marcus, The Spectrum of the $S^{5}$ Compactification of the Chiral $N=2$, $D=10$ Supergravity and the Unitary Supermultiplets of U(2, 2/4), Class. Quant. Grav. 2 (1985) L11 [INSPIRE].

[68] M. Günaydin and D. Volin, The complete unitary dual of non-compact Lie superalgebra su $(p, q \mid m)$ via the generalised oscillator formalism and non-compact Young diagrams, arXiv: 1712.01811 [INSPIRE].

[69] N. Kanning, Y. Ko and M. Staudacher, Graßmannian integrals as matrix models for non-compact Yangian invariants, Nucl. Phys. B 894 (2015) 407 [arXiv:1412.8476] [INSPIRE].

[70] H. Leutwyler and A.V. Smilga, Spectrum of Dirac operator and role of winding number in QCD, Phys. Rev. D 46 (1992) 5607 [inSPIRE].

[71] D.J. Gross and E. Witten, Possible Third Order Phase Transition in the Large $N$ Lattice Gauge Theory, Phys. Rev. D 21 (1980) 446 [inSPIRE].

[72] E. Brézin and D.J. Gross, The External Field Problem in the Large N Limit of QCD, Phys. Lett. 97B (1980) 120 [INSPIRE].

[73] I. Bars and F. Green, Complete Integration of $U(N)$ Lattice Gauge Theory in a Large $N$ Limit, Phys. Rev. D 20 (1979) 3311 [InSPIRE].

[74] B. Schlittgen and T. Wettig, Generalizations of some integrals over the unitary group, J. Phys. A 36 (2003) 3195 [math-ph/0209030] [INSPIRE].

[75] A.B. Balantekin, Character expansions, Itzykson-Zuber integrals and the QCD partition function, Phys. Rev. D 62 (2000) 085017 [hep-th/0007161] [INSPIRE].

[76] B. Eden and M. Staudacher, Integrability and transcendentality, J. Stat. Mech. 0611 (2006) P11014 [hep-th/0603157] [INSPIRE].

[77] N. Beisert, B. Eden and M. Staudacher, Transcendentality and Crossing, J. Stat. Mech. 0701 (2007) P01021 [hep-th/0610251] [INSPIRE].

[78] A. Mironov, A. Morozov and G.W. Semenoff, Unitary matrix integrals in the framework of generalized Kontsevich model. 1. Brezin-Gross-Witten model, Int. J. Mod. Phys. A 11 (1996) 5031 [hep-th/9404005] [INSPIRE].

[79] A.Y. Orlov, New Solvable Matrix Integrals, Int. J. Mod. Phys. A 19 (2004) 276 [nlin/0209063].

[80] T. Miwa, M. Jimbo and E. Date, Solitons: Differential Equations, Symmetries and Infinite Dimensional Algebras, Cambridge University Press, Cambridge U.K. (2000).

[81] V. Bargmann, On a Hilbert Space of Analytic Functions and an Associated Integral Transform Part I, Commun. Pure Appl. Math. 14 (1961) 187.

[82] L.A. Takhtajan, Quantum Mechanics for Mathematicians, American Mathematical Society, Providence U.S.A. (2008).

[83] J. Zinn-Justin, Path Integrals in Quantum Mechanics, Oxford University Press, Oxford U.K. (2005).

[84] H. Boos, F. Göhmann, A. Klümper, K.S. Nirov and A.V. Razumov, Exercises with the universal R-matrix, J. Phys. A 43 (2010) 415208 [arXiv: 1004.5342] [INSPIRE]. 
[85] B.S. Shastry, Exact Integrability of the One-Dimensional Hubbard Model, Phys. Rev. Lett. 56 (1986) 2453 [INSPIRE].

[86] C. Gómez, M. Ruiz-Altaba and G. Sierra, Quantum Groups in Two-Dimensional Physics, Cambridge University Press, Cambridge U.K. (2005).

[87] P.D. Miller, Applied Asymptotic Analysis, American Mathematical Society, Providence U.S.A. (2006).

[88] Z. Bern, L.J. Dixon and V.A. Smirnov, Iteration of planar amplitudes in maximally supersymmetric Yang-Mills theory at three loops and beyond, Phys. Rev. D 72 (2005) 085001 [hep-th/0505205] [INSPIRE].

[89] Z. Bern et al., The Two-Loop Six-Gluon MHV Amplitude in Maximally Supersymmetric Yang-Mills Theory, Phys. Rev. D 78 (2008) 045007 [arXiv: 0803.1465] [INSPIRE].

[90] A. Hurwitz, Über die Erzeugung der Invarianten durch Integration, Gesell. Wiss. Göttingen Nachr. Math-Phys. Kl. 1897 (1897) 71, http://eudml.org/doc/58378.

[91] R. Gilmore, Lie Groups, Lie Algebras, and Some of Their Applications, John Wiley \& Sons, New York U.S.A. (1974). 